Article

\title{
Microfluidic Airborne Metal Particle Sensor Using Oil Microcirculation for Real-Time and Continuous Monitoring of Metal Particle Emission
}

\author{
Jong-Seo Yoon, Jiwon Park, Hye-Rin Ahn, Seong-Jae Yoo and Yong-Jun Kim * \\ School of Mechanical Engineering, Yonsei University, 50 Yonsei-ro, Seodaemun-gu, Seoul 03722, Korea; \\ jsyoon129@yonsei.ac.kr (J.-S.Y.); prion1548@yonsei.ac.kr (J.P.); hyerinahn@yonsei.ac.kr (H.-R.A.); \\ sj92@yonsei.ac.kr (S.-J.Y.) \\ * Correspondence: yjk@yonsei.ac.kr; Tel.: +82-2-2123-7212
}

Citation: Yoon, J.-S.; Park, J.; Ahn, H.-R.; Yoo, S.-J.; Kim, Y.-J.

Microfluidic Airborne Metal Particle Sensor Using Oil Microcirculation for Real-Time and Continuous

Monitoring of Metal Particle

Emission. Micromachines 2021, 12, 825 . https://doi.org/10.3390/mi12070825

Academic Editor: Dimitris Tsoukalas

Received: 5 June 2021

Accepted: 11 July 2021

Published: 14 July 2021

Publisher's Note: MDPI stays neutral with regard to jurisdictional claims in published maps and institutional affiliations.

Copyright: (c) 2021 by the authors. Licensee MDPI, Basel, Switzerland. This article is an open access article distributed under the terms and conditions of the Creative Commons Attribution (CC BY) license (https:/ / creativecommons.org/licenses/by/ $4.0 /)$.

\begin{abstract}
Airborne metal particles (MPs; particle size $>10 \mu \mathrm{m}$ ) in workplaces result in a loss in production yield if not detected in time. The demand for compact and cost-efficient MP sensors to monitor airborne MP generation is increasing. However, contemporary instruments and laboratorygrade sensors exhibit certain limitations in real-time and on-site monitoring of airborne MPs. This paper presents a microfluidic MP detection chip to address these limitations. By combining the proposed system with microcirculation-based particle-to-liquid collection and a capacitive sensing method, the continuous detection of airborne MPs can be achieved. A few microfabrication processes were realized, resulting in a compact system, which can be easily replaced after contamination with a low-priced microfluidic chip. In our experiments, the frequency-dependent capacitive changes were characterized using MP (aluminum) samples (sizes ranging from $10 \mu \mathrm{m}$ to $40 \mu \mathrm{m}$ ). Performance evaluation of the proposed system under test-bed conditions indicated that it is capable of real-time and continuous monitoring of airborne MPs (minimum size $10 \mu \mathrm{m}$ ) under an optimal frequency, with superior sensitivity and responsivity. Therefore, the proposed system can be used as an on-site MP sensor for unexpected airborne MP generation in precise manufacturing facilities where metal sources are used.
\end{abstract}

Keywords: microfluidics; microcirculation; particle-to-liquid collection; airborne metal particle; continuous and real-time monitoring; capacitive detection

\section{Introduction}

The development of automotive technologies has been rapidly expanding owing to an increased focus on reducing environmental impact [1,2]. Various electronics have been embedded into autonomous systems for different operations, such as supplying power and controlling steering systems [3]. Following this ongoing trend regarding driving devices, there is a demand for the miniaturization and integration of electronics to achieve spatial savings, low power consumption, and high electrical performance [4,5]. For packaged semiconductor chips, rechargeable batteries, and other components that aim at the same goal, greater safety and quality are essential [6]. However, tremendous effort should be invested because these electronic components have a wide range of production failures [7]. Among the common issues, airborne metal particles (MPs; particles larger than $10 \mu \mathrm{m}$ ) at manufacturing sites are one of the root causes of the degradation of the lifespan and reliability of the products, because they induce internal short circuits or stress points $[8,9]$. There have been advances in manufacturing procedures such as encapsulation packaging, electrode welding, and cutting; however, micrometer-sized MPs break down from the metal sources, scatter in the air, and settle on multiple spots that may be electrically connected [10,11]. In addition, when the deterioration of manufacturing machinery begins, the numbers and sizes of MPs become larger [12,13]. Thus, airborne MPs must be monitored to improve the current production yield. 
Currently, compositional analysis instruments have been widely adopted to determine the level of contamination and particle composition using an X-ray and inductively coupled plasma techniques [14-16]. The samples used for analysis must be collected in advance and pre-treated. Adhesive films are placed at points of potential emitters in manufacturing lines and are routinely replaced [17]. Manufacturers can obtain quantitative (i.e., number and size) and qualitative (i.e., element map) information through sample analysis [18]. Although the current analytical system can determine the approximate contamination without temporal resolution, the procedures are time-consuming and complex. Moreover, these instruments are expensive, have a large volumetric size, and require regular maintenance, which leads to limitations in monitoring the MPs and the points of interest on a real-time basis. Thus, inexpensive compact sensors are required for on-site monitoring and to establish a dense monitoring network throughout the worksites.

Instead of monitoring MPs in the air, many studies have been performed to detect MPs in a hydraulic oil [12]. These attempts have been based on electrical detection, such as inductive or capacitive techniques [19-28]. Most contemporary laboratory-grade sensors have successfully detected MPs in sizes ranging from meso- to micro-scale in real-time. Among them, a sensor based on a capacitive detection technique was developed based on a microfluidic platform with a greater sensitivity than the inductive method [28]. They demonstrated real-time monitoring of MPs up to $10 \mu \mathrm{m}$ with a micro-sized sensing volume. However, previous studies have only focused on detecting MPs in hydraulic oil without a particle collection system. To monitor airborne MPs, though they are not easily detected in oil, a microfluidic electrical detection system with a particle collection system should be devised.

However, it is difficult to directly measure airborne MPs owing to their heavy gravity. Moreover, cumulative particle detection, which identifies the increasing steps of signals depending on particle accumulation, is not an approved method for monitoring MPs [29,30]. This detection mechanism can cause short circuits on electrical detection elements and hence, require periodic cleaning. To pass the particles over the detection element, a viscous liquid as a particle carrier fluid is essential. Hence, because a sensing element is in the liquid phase, a particle-to-liquid collection method must be incorporated. Several approaches have been proposed, and many studies in the field of bioaerosol analysis have shown continuous monitoring based on this collection method [31,32]. Despite the considerable progress, they are currently not applicable for monitoring airborne MPs because they are bulky and complicated. This is because their liquid supply and exhaust system, which exist independently, occupy a lot of space to maintain a liquid surface for particle inflow. Therefore, an integrated microfluidic system with circulation-based particle delivery from the air to the liquid and electrical detection is required for the continuous and sensitive monitoring of MPs.

Herein, we present the integration of a microfluidic channel and a capacitive particle detection element into a single chip that can detect airborne MPs continuously in real-time. The proposed system is composed of a microcirculation-based particle delivery system and a particle detection element. Airborne MPs settle in the liquid and are delivered to the detection region by microcirculation. A coplanar capacitive sensor used for particle detection was adopted owing to its high sensitivity and simple fabrication with a microchannel. Microfabricated interdigitated electrodes (IDEs) with a coplanar configuration were realized at the bottom of the relatively small points in the microchannel. The capacitive change generated by individual particles greatly depends on the dielectric properties, particle size, particle $z$-axis position from the electrodes, and the applied AC voltage. By adopting hydraulic oil as the particle carrier fluid, we obtained the advantages of particle detection and delivery. Furthermore, the oil reservoir plays the role of a tank for circulation; however, it is also a place where the detected particles are sunk or accumulated. If the MP detection chip is highly contaminated by particles after operating for a long time, it can be disposed and replaced. Our chip is low in cost because it is fabricated in a batch process and easily assembled with other microelectromechanical system (MEMS)-compatible parts. Thus, 
we demonstrate the feasibility of monitoring airborne MPs by integrating these novel functions. Our system is expected to be widely applicable in workplaces that must deal with metal sources, because it facilitates immediate action and determines the causes of defect points.

\section{Principle of Particle Detection}

The capacitive sensing technique on a microfluidic platform to measure the variations generated by particles is mainly based on the contrast of dielectric properties (e.g., permittivity and conductivity). By placing the electrodes close to the particle matter trajectory, the particles passing through the detection region are inevitably affected by an AC electrical field. Based on this phenomenon, such variations are directly related to the interaction of the created electric field as well as the occupied volume fraction between the matter and medium in the detection region. Therefore, in this study, the presence of MPs is displaced by the equivalent capacitance and measured as a positive pulse, as illustrated in Figure 1. Our particle detection element was performed using an LCR meter, which is a high-precision instrument, rather than a lock-in amplifier, owing to its interface convenience. The capacitance variations can be equivalently calculated by measuring the impedance of the capacitor.
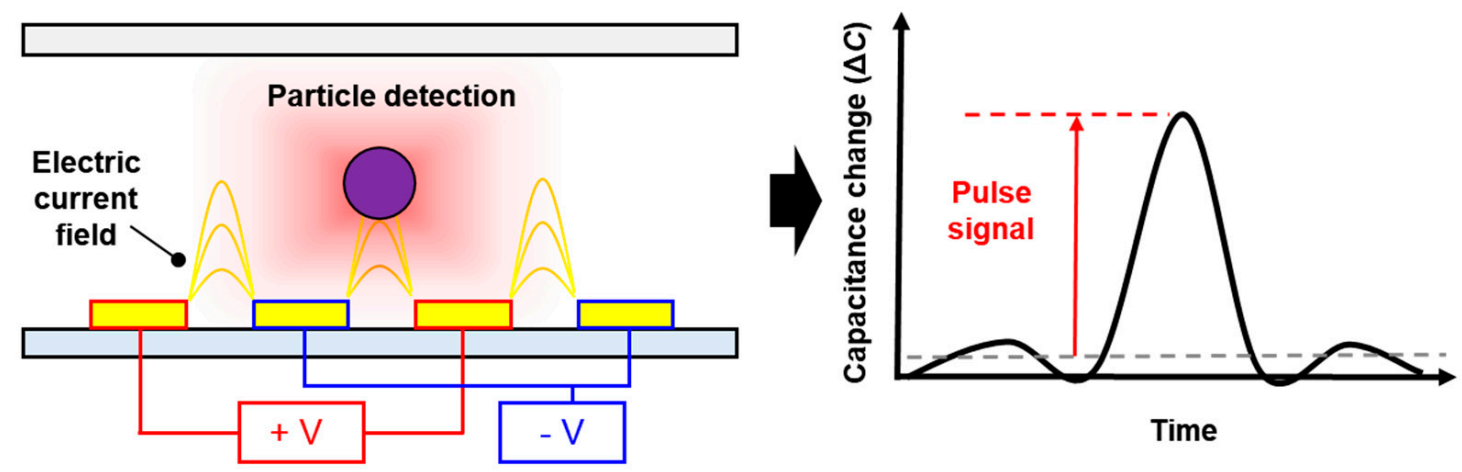

Figure 1. Simplified schematic diagram for particle detection under applied AC voltage.

Impedance $(Z)$ is defined as the ratio of two electrical characteristics, one of which depends on variations in the dielectric properties (permittivity and conductivity) of the system (i.e., electric current response (I)), and the other is the frequency-dependent excitation signal with a small voltage (AC voltage $(\mathrm{V}))$ [33].

$$
Z(j \omega)=\frac{V(j \omega)}{I(j \omega)}=Z_{r e}+j Z_{i m}
$$

where $j^{2}=-1, \omega$ is the angular frequency; $Z_{r e}$ is the real part (resistance), and $Z_{i m}$ is the imaginary part (conductance) of the complex impedance.

Based on the above equation, according to the principle of the specific mode in the LCR meter used in this study, the impedance is calculated as follows [34]:

$$
\mathrm{Z}=\frac{\mathrm{R} X_{C^{2}}}{\mathrm{R}^{2}+\mathrm{X}_{\mathrm{C}}{ }^{2}}+\mathrm{j} \frac{\mathrm{R}^{2} \mathrm{X}_{\mathrm{C}}}{\mathrm{R}^{2}+\mathrm{X}_{\mathrm{C}}{ }^{2}}
$$

where $R$ is the resistance, and $X_{C}$ is the capacitive reactance. $X_{C}$ is given as follows:

$$
\mathrm{X}_{\mathrm{C}}=\frac{1}{j \omega \mathrm{C}_{\mathrm{eq}}}
$$


Therefore, the equivalent capacitance $\left(\mathrm{C}_{\mathrm{eq}}\right)$ can be derived as follows:

$$
\mathrm{C}_{\mathrm{eq}}=\frac{1}{\omega} \cdot \frac{\mathrm{Z}_{\mathrm{im}}}{\left(\mathrm{Z}_{\mathrm{re}}\right)^{2}+\left(\mathrm{Z}_{\mathrm{im}}\right)^{2}}
$$

A simplified electrical equivalent circuit model is presented in Figure 2 to illustrate the electrical variations in the detection region. The measured impedance $\left(\mathrm{Z}_{\text {total }}\right)$ can be expressed as a part of capacitance $(C)$ and resistance $(R)$ in parallel. It includes the capacitance $\left(C_{d r}\right)$ and resistance $\left(R_{d r}\right)$ around the electrodes in the detection region. The measured impedance is expressed as follows:

$$
\mathrm{Z}_{\mathrm{total}}=\mathrm{R}_{\mathrm{dr}}+\frac{1}{\mathrm{j \omega} \mathrm{C}_{\mathrm{dr}}}
$$

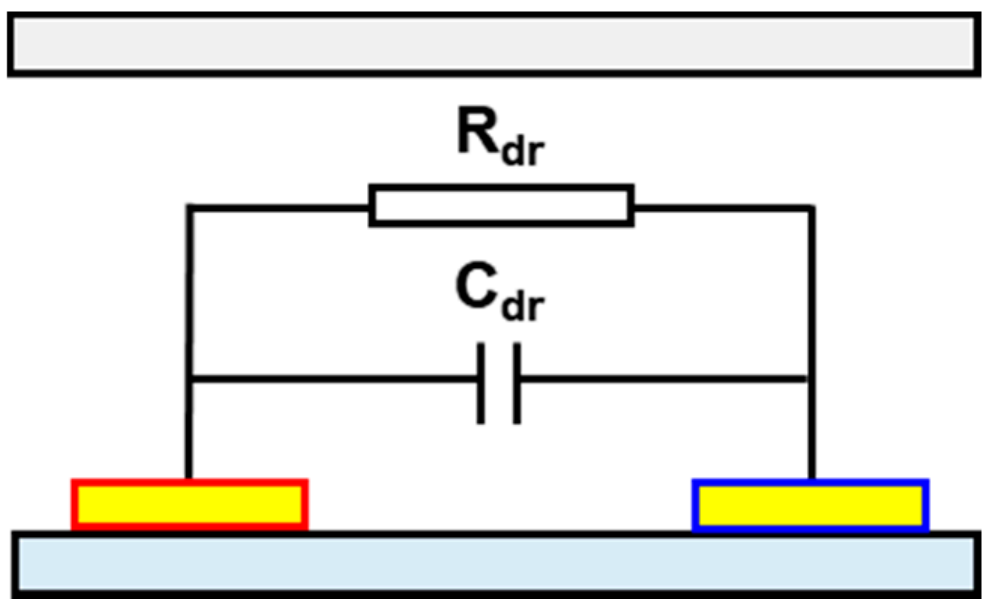

Figure 2. Equivalent electrical circuit of the detection region.

In practice, the dielectric behavior varies depending on the operating conditions. The permittivity is given as follows:

$$
\widetilde{\varepsilon}=\varepsilon-j \frac{\sigma}{\omega}
$$

where $\widetilde{\varepsilon}$ is the complex permittivity, and $\sigma$ is the conductivity.

Therefore, the impedance $\left(Z_{0}\right)$ of the capacitor in the empty site, which is filled with the medium, is given as follows:

$$
\mathrm{Z}_{0}=\frac{1}{j \omega \widetilde{\varepsilon}_{\mathrm{med}} \mathrm{G}_{\mathrm{f}}}
$$

where $\widetilde{\varepsilon}_{\text {med }}$ is the complex permittivity of the medium, and $\mathrm{G}_{\mathrm{f}}$ is the geometric constant based on the configuration of the electrodes. In this study, we adopted an interdigital shape for the electrode configuration.

In the case of a particle in the medium, the equivalent complex permittivity $\left(\widetilde{\varepsilon}_{\text {mix }}\right)$ is well described using the Maxwell mixture theory because of the interaction between the particles and the medium [35].

$$
\widetilde{\varepsilon}_{\text {mix }}=\widetilde{\varepsilon}_{\text {med }} \frac{1+2 \Phi \widetilde{\mathrm{f}}_{\mathrm{CM}}}{1-\Phi \widetilde{\mathrm{f}}_{\mathrm{CM}}}
$$

where $\Phi$ is the volume fraction, and $\widetilde{\mathrm{f}}_{\mathrm{CM}}$ is the Clausius-Mossotti factor. $\Phi$ is the volume fraction of the occupied volume of the particles $\left(\mathrm{V}_{\mathrm{ptc}}\right)$ and the detection region $\left(\mathrm{V}_{\mathrm{dr}}\right)$ and is defined as

$$
\Phi=\frac{\mathrm{V}_{\mathrm{ptc}}}{\mathrm{V}_{\mathrm{dr}}}
$$


and $\widetilde{\mathrm{f}}_{\mathrm{CM}}$ is the Clausius-Mossotti formula of the dielectric function defined as follows:

$$
\widetilde{\mathrm{f}}_{\mathrm{CM}}=\frac{\widetilde{\varepsilon}_{\text {ptc }}-2 \widetilde{\varepsilon}_{\text {med }}}{\widetilde{\varepsilon}_{\text {ptc }}+2 \widetilde{\varepsilon}_{\text {med }}}
$$

From (8)-(10), $\widetilde{\varepsilon}_{\text {mix }}$ is derived as follows:

$$
\widetilde{\varepsilon}_{\text {mix }}=\widetilde{\varepsilon}_{\text {med }} \frac{\mathrm{V}_{\mathrm{dr}}\left(\widetilde{\varepsilon}_{\mathrm{ptc}}+2 \widetilde{\varepsilon}_{\text {med }}\right)+\mathrm{V}_{\mathrm{ptc}}\left(\widetilde{\varepsilon}_{\mathrm{ptc}}-\widetilde{\varepsilon}_{\text {med }}\right)}{V_{\mathrm{dr}}\left(\widetilde{\varepsilon}_{\mathrm{ptc}}+2 \widetilde{\varepsilon}_{\text {med }}\right)-\mathrm{V}_{\mathrm{ptc}}\left(\widetilde{\varepsilon}_{\mathrm{ptc}}-\widetilde{\varepsilon}_{\text {med }}\right)}
$$

Therefore, the impedance $\left(Z_{\text {mix }}\right)$ of the capacitor in the medium containing the particle is given as follows:

$$
\mathrm{Z}_{\text {mix }}=\frac{1}{j \omega \widetilde{\varepsilon}_{\text {mix }} G_{f}}
$$

Combining the above equations, the equivalent capacitance variation $\left(\Delta \mathrm{C}_{\mathrm{eq}}\right)$ can be obtained as follows:

$$
\Delta \mathrm{C}_{\mathrm{eq}}=\mathrm{C}_{\mathrm{eq}, \mathrm{mix}}-\mathrm{C}_{\mathrm{eq}, \mathrm{med}}=\frac{1}{\omega} \cdot\left\{\frac{\mathrm{Z}_{\text {mix,im }}}{\left(Z_{\text {mix }, \mathrm{re}}\right)^{2}+\left(\mathrm{Z}_{\mathrm{mix}, \mathrm{im}}\right)^{2}}-\frac{\mathrm{Z}_{\mathrm{med}, \mathrm{im}}}{\left(\mathrm{Z}_{\text {med,re }}\right)^{2}+\left(\mathrm{Z}_{\text {med,im }}\right)^{2}}\right\}
$$

The amplitude of the capacitive pulse signal $\left(\Delta \mathrm{C}_{\mathrm{eq}}\right)$ depends on the excitation frequency $(\omega)$, occupied volume of the particles $\left(\mathrm{V}_{\mathrm{ptc}}\right)$, and mixture complex permittivity $\left(\widetilde{\varepsilon}_{\text {mix }}\right)$. Based on Equation (9), the sensing volume is crucial, along with the particle size to be detected. This is because the signal $\left(\Delta \mathrm{C}_{\mathrm{eq}}\right)$ can be increased accordingly if the particles occupy a larger volumetric proportion. Based on Equation (13), although increasing the frequency lowers the capacitance change and the noise floor, it is effective for distinguishing the frequency-dependent capacitance change in metals [36]. This is because the particles may be measured similarly to the baseline level of noise at low frequencies. Therefore, it is essential to optimize the operating conditions and geometrical factors of the structure (microfluidic channel and IDEs) to enhance the sensing performance.

\section{Sensor Design and Fabrication}

\subsection{Operating Principle}

Our microfluidic sensor has two components: (a) an automated particle delivery system and (b) a particle detection element as shown in Figure 3. Airborne MPs are settled down to the funnel-shaped structures because of gravity. The particles drawn into the microchannel because of gravity or air outflow as vacuum air. The particles are impacted into the liquid and lose their velocity owing to the viscosity of the working fluid. Herein, the exhaust path exists to prevent oil overflow due to problems with particle clogging and pump pulsation. They are delivered to the particle detection region by microcirculation. For the detection region, the microfabricated IDEs used as the particle detection element perform the electrical excitation of the entire sensing volume and measure the electrical response in real-time. Subsequently, the particles pass through the detection region sink by escaping the mainstream of the microcirculation owing to their inertia and accumulating at the bottom of the oil reservoir. 


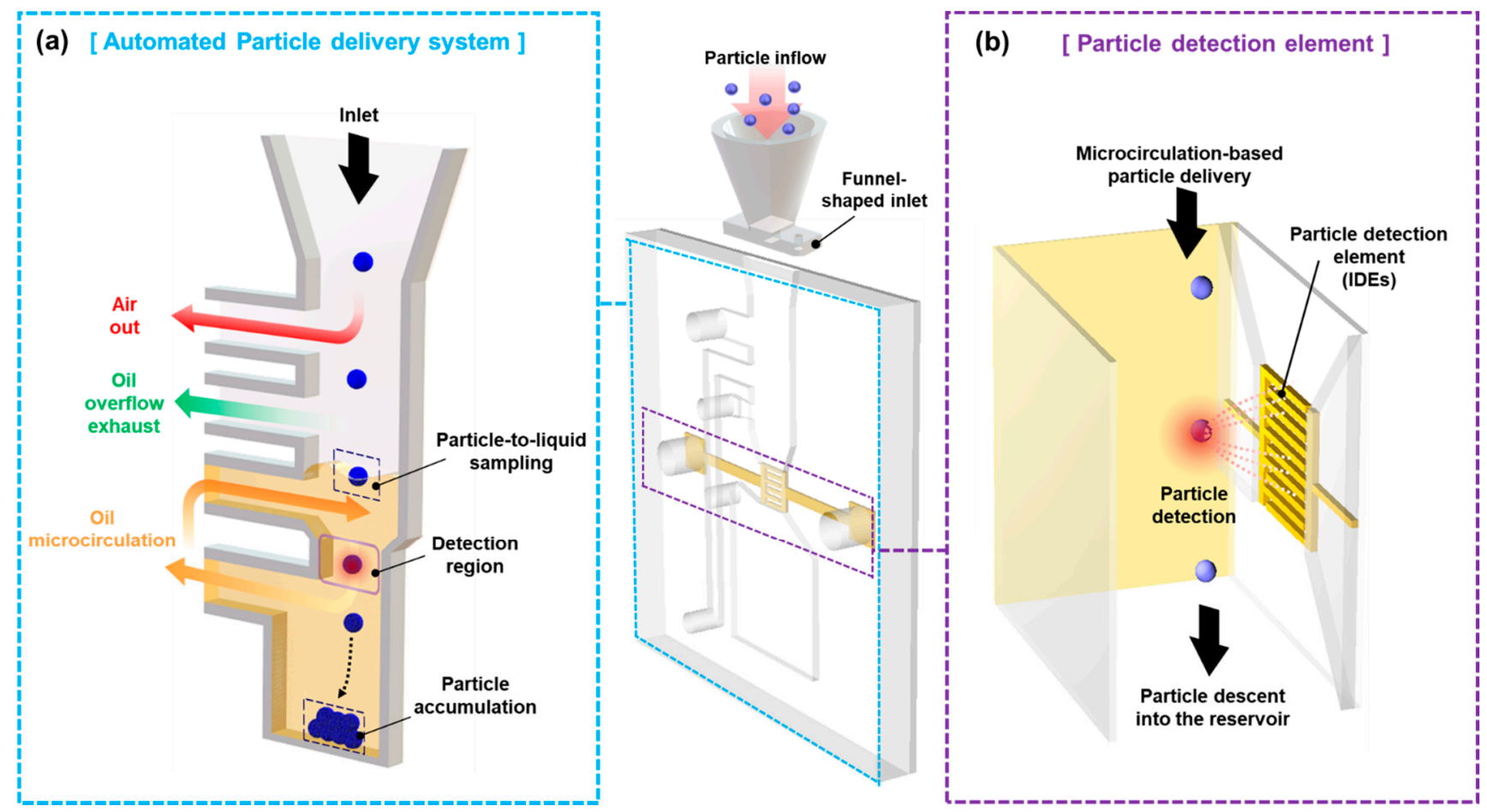

Figure 3. Schematic illustration of the proposed system. Our system consists of two sections: (a) an automated particle delivery system and (b) a particle detection element.

\subsection{Design of Automated Particle Delivery System}

A funnel-shaped structure was assembled in front of our microfluidic chip to induce airborne MPs toward the small opening of our microfluidic chip. This part helps in collecting scattered and free-falling particles with a wide geometrical design. The microfluidic chip connected to this structure was realized to perform two main roles for automated and continuous particle delivery: (i) collecting the particles that enter from the air to the viscous liquid by maintaining the liquid surface on the chip and (ii) delivering these particles to the particle detection element by internal microcirculation.

Hydraulic oil was adopted as the particle carrier liquid because it has a sufficient density $\left(0.8 \times 10^{3} \mathrm{~kg} / \mathrm{m}^{3}\right)$ to deliver the MPs (e.g., aluminum is $\left.2.9 \times 10^{3} \mathrm{~kg} / \mathrm{m}^{3}\right)$ and has a low-conductive media (the relative permittivity of the oil $\varepsilon_{0}$ is 2.6) for visible dielectric contrast against the metals. The use of oil in a microchannel has already been validated as MPs are transported adequately to their detection elements [12]. Here, the MPs in chip operation are mainly influenced by the wall-induced lift force, mainstream drag force, and gravitational force $[37,38]$. Among them, the drag force and gravity owing to the dynamic viscosity of the oil and heavy gravity of the metal, respectively, have a dominant influence on the particle motion. Thus, our microfluidic device was placed at an angle to the ground to match the vectors of gravity and the mainstream toward the detection region. Through this placement, we could avoid unnecessary possibilities that could lead to chip failures in microfluidics, such as particle clogging or bottlenecks, and induce the MPs to go down to the oil reservoir section [39].

For stable microcirculation of the viscous liquid (oil), we designed the overall structure of the microchannel to be rectangular in shape with a low aspect ratio $(6000 \mu \mathrm{m} \times 500 \mu \mathrm{m})$ [40]. Based on the geometrical structure with margins, it allows precise flow control and enhances the stable operation. As an exception, only the dimensions of the required points (detection region and inlet of the oil microcirculatory system) were determined to be relatively low $(2000 \mu \mathrm{m} \times 60 \mu \mathrm{m} \times 400 \mu \mathrm{m})$, as illustrated in Figure 4. Of the two points, the detection region was made to allow the particles to pass through closely to the particle detection element, and it occupied a large fraction of the sensing volume [41]. The other was 
matched with the dimensions of the detection region to minimize excessive mismatches in the flow rate of the microcirculation between the oil inlet and outlet. For each entrance line, the structure was designed in a tapered shape to avoid bottlenecks. It induces the incoming particles to the detection region to accelerate and pass through quickly. Because this microchannel is MEMS-compatible and easily fabricated, synergies can be obtained depending on the architecture of the microfabricated electrodes.

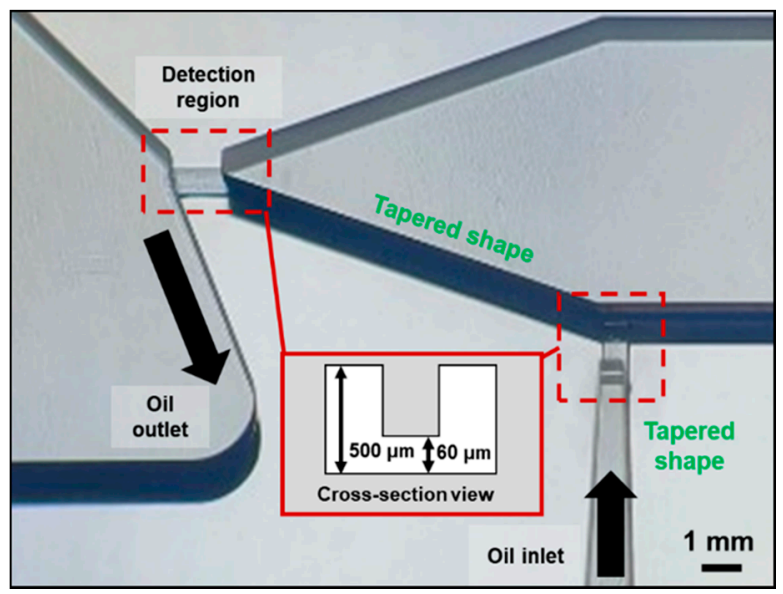

Figure 4. Optical photograph of the relatively small volumetric points (detection region and oil inlet) on the SU-8 mold.

\subsection{Design of Particle Detection Element}

\subsubsection{Planar Electrode Array}

The electrode configuration for creating an electric field has been mainly adopted as parallel or planar in particle detection sensor applications. Because electric current lines interact with the presence of matter in the media, a denser electric field is required. In the case of parallel electrodes, the distance between the electrodes is a key parameter because of the homogenous electric field [42]. Although a smaller gap could enhance the sensing performance, its geometrical dimension depends on the largest size of the particles to avoid such problems as particle clogging. Moreover, the fabrication procedures are complex because the electrodes are mechanically integrated at microscale intervals. Conversely, the other geometry is relatively simple because it can be fabricated on the same plane. In addition, the planar type exhibits higher sensing synergies with a microfluidic channel [43]. Owing to its non-uniform electric field, the peak signal becomes larger as the particles move closer toward the electrodes. In other words, the planar model could have the advantage of being able to detect small-sized particles with a more concentrated high-intensity electric field than the parallel one. Thus, the planar electrode array is a suitable configuration for our microfluidic platform.

\subsubsection{Interdigitated Microelectrodes}

Various studies have attempted to determine the optimal dimensions of electrodes using finite-element simulations validating that the shorter the distance between the electrodes, the larger the electric variations produced by the particles $[30,44-46]$. This is because the electric field line creates a strong boundary between the electrodes. Although the effect of geometrical optimization tends to be saturated, the order of the measured values changes considerably in terms of performance enhancement. For our microfluidic platform, the geometric design factors of planar electrodes need to be determined with data sampling frequency and particle residence time to detect the particles passing through the detection region in a short time. The design values of the required sensing area can be theoretically derived based on the determined flow rate of the working fluid and the geometric structure of the microchannel. Here, simply extending the distance between the electrodes would lower the intensity of the electric field. A single pair of coplanar 
electrodes could not sufficiently detect the particle behavior without increasing the applied voltage. Therefore, as illustrated in Figure 5, we adopted a shape for the electrodes, namely, several interdigitated pairs. The finger gap $(\mathrm{g})$ and width $(\mathrm{w})$ of the IDEs were 20 and $40 \mu \mathrm{m}$, respectively. They are the minimum linewidth and gap that can be achieved with the conventional lithography process using the film mask we have used. The finger length (l) was $390 \mu \mathrm{m}$. The width of the detection region and the finger length was matched so that the particle was affected by the electric field as soon as the particle enters from the entrance of the detection region. The number of fingers (n) was 13, chosen to ensure a sufficient sensing area. To detect at least two points or more with the sampling rate while the particle passes through the sensing volume, the sensing area was expanded with several pairs of fingers. The thickness of the electrodes were determined to be $0.21 \mu \mathrm{m}$ to prevent unexpected bonding issues owing to the high flush between the microelectrodes and the microchannel [47]. Accordingly, the design values of the total electrode length $\left(T_{L}\right)$ and width $\left(\mathrm{T}_{\mathrm{W}}\right)$ were $1480 \mu \mathrm{m}$ and $490 \mu \mathrm{m}$, respectively. The design factors of the IDE are listed in Table 1.

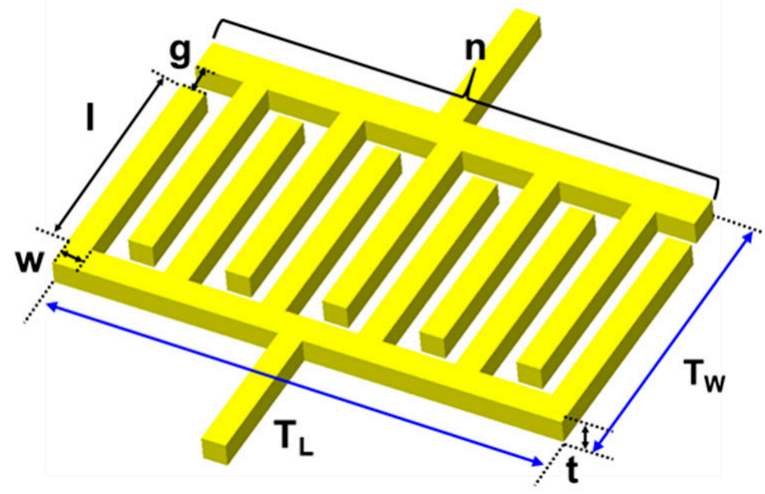

Figure 5. Simplified view of interdigitated electrodes with defined design factors.

Table 1. Design factors of the IDEs.

\begin{tabular}{cc}
\hline Design Factors & Value \\
\hline Finger gap (g) & $20 \mu \mathrm{m}$ \\
Finger width (w) & $40 \mu \mathrm{m}$ \\
Finger length (l) & $390 \mu \mathrm{m}$ \\
Number of fingers $(\mathrm{n})$ & 13 \\
Thickness $(\mathrm{t})$ & $0.21 \mu \mathrm{m}$ \\
Total length $\left(\mathrm{T}_{\mathrm{L}}\right)$ & $1480 \mu \mathrm{m}$ \\
Total width $\left(\mathrm{T}_{\mathrm{w}}\right)$ & $490 \mu \mathrm{m}$ \\
\hline
\end{tabular}

\subsection{Sensor Fabrication}

Figure 6a presents photographs of the proposed packaged system. The prototype is compact $(64 \mathrm{~mm} \times 120 \mathrm{~mm} \times 12 \mathrm{~mm}$ ) for the on-site monitoring of airborne MPs at the points of interest. Using a semiconductor fabrication process, the microfluidic MP detection chip was realized on a single chip $(52.5 \mathrm{~mm} \times 52.5 \mathrm{~mm} \times 7 \mathrm{~mm})$, as presented in Figure $6 \mathrm{~b}$. The IDEs as particle detection elements could be identified and were placed in the detection region where the particles passed through, as presented in Figure 6c. 


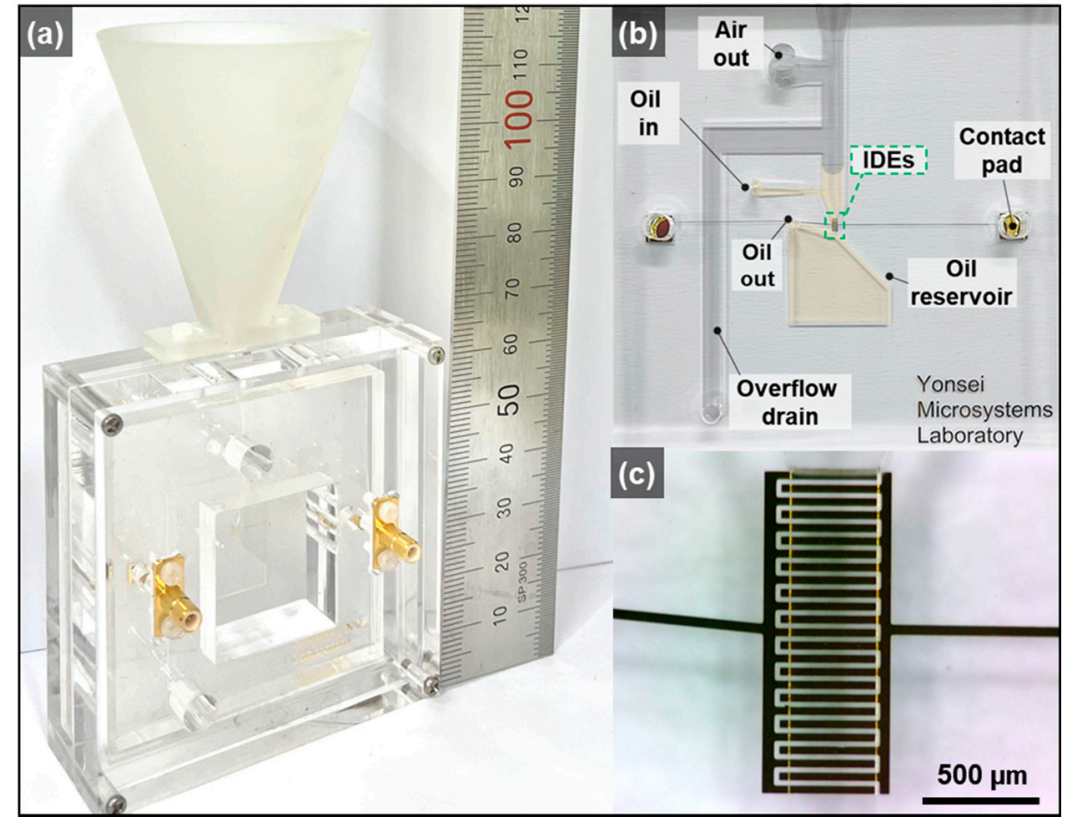

Figure 6. Optical paragraphs of (a) the proposed packaged system, (b) the microfluidic MP detection chip, and (c) the microfabricated interdigitated electrodes as a particle detection element.

Figure 7 illustrates the fabrication processes, which is explained in two schematic diagrams: (i) the microfluidic MP detection chip and (ii) the packaging process. IDEs were fabricated using photolithography. First, we deposited a thin metal layer (40/170 nm of $\mathrm{Cr} / \mathrm{Au}$ ) on a glass slide using an electron-beam evaporator. Subsequently, we spin-coated the positive-tone photoresist (AZ GXR 601, AZ Electronic Materials Co., Ltd., Wilmington, DE, USA) for $30 \mathrm{~s}$ at $3000 \mathrm{rpm}$ and baked it softly on a hot plate for $1 \mathrm{~min}$ at $85{ }^{\circ} \mathrm{C}$. After the glass was cooled to $25^{\circ} \mathrm{C}$, it was exposed to UV light with a printed film mask and was developed with a photoresist developer. To pattern the IDEs, wet etching was performed, following each etchant of the deposition layer in sequence (Figure 7a). In the case of fabricating the microchannel, the SU-8 negative tone photoresist, which has been extensively used in microfluidics, was adopted with a difference in height $(500 / 60 \mu \mathrm{m})$. The first layer was coated (60 $\mu \mathrm{m}$, model SU 8-50, Microchem Co., Westborough, MA, USA) on a Si wafer for $30 \mathrm{~s}$ at $1800 \mathrm{rpm}$ and heated gently at two different temperatures $\left(65^{\circ} \mathrm{C}\right.$ for $6 \mathrm{~min}$ and $95^{\circ} \mathrm{C}$ for $20 \mathrm{~min}$ ). Thereafter, it was exposed to UV light to define a relatively small aperture for the detection region and oil inlet. Because it was difficult to define a $500 \mu \mathrm{m}$ layer at once, we proceeded with the deposition twice. The $250 \mu \mathrm{m}$ layer was coated for $30 \mathrm{~s}$ at $1000 \mathrm{rpm}$, and a soft bake was conducted $\left(65^{\circ} \mathrm{C}\right.$ for $30 \mathrm{~min}$ and $95^{\circ} \mathrm{C}$ for $90 \mathrm{~min}$ ). Next, the last $250 \mu \mathrm{m}$ layer was formed in the same way as the first layer. After exposure to UV light, the baking process was conducted at $65^{\circ} \mathrm{C}$ for $1 \mathrm{~min}$ and $95^{\circ} \mathrm{C}$ for $40 \mathrm{~min}$. Both exposed layers were developed using an SU-8 developer (Figure $7 \mathrm{~b}$ ). To realize the microchannel, Sylgard 184 (Dow Silicones Co., Midland, MI, USA) was mixed in advance at a standard cross-linker ratio of 10:1. Polydimethylsiloxane (PDMS) was cast to a thickness of $6.5 \mathrm{~mm}$ and placed in an environmental chamber for thermal aging $\left(60{ }^{\circ} \mathrm{C}\right.$ for $6 \mathrm{~h}$ ). The PDMS was then peeled and punched using a biopsy (Figure $7 \mathrm{c}$ ). To integrate the microchannel with a gold-patterned glass, the microchannel was exposed to oxygen plasma. Subsequently, an irreversible seal was formed by contacting the oxidized microchannel with an accurate alignment to the glass surface (Figure 7d). 

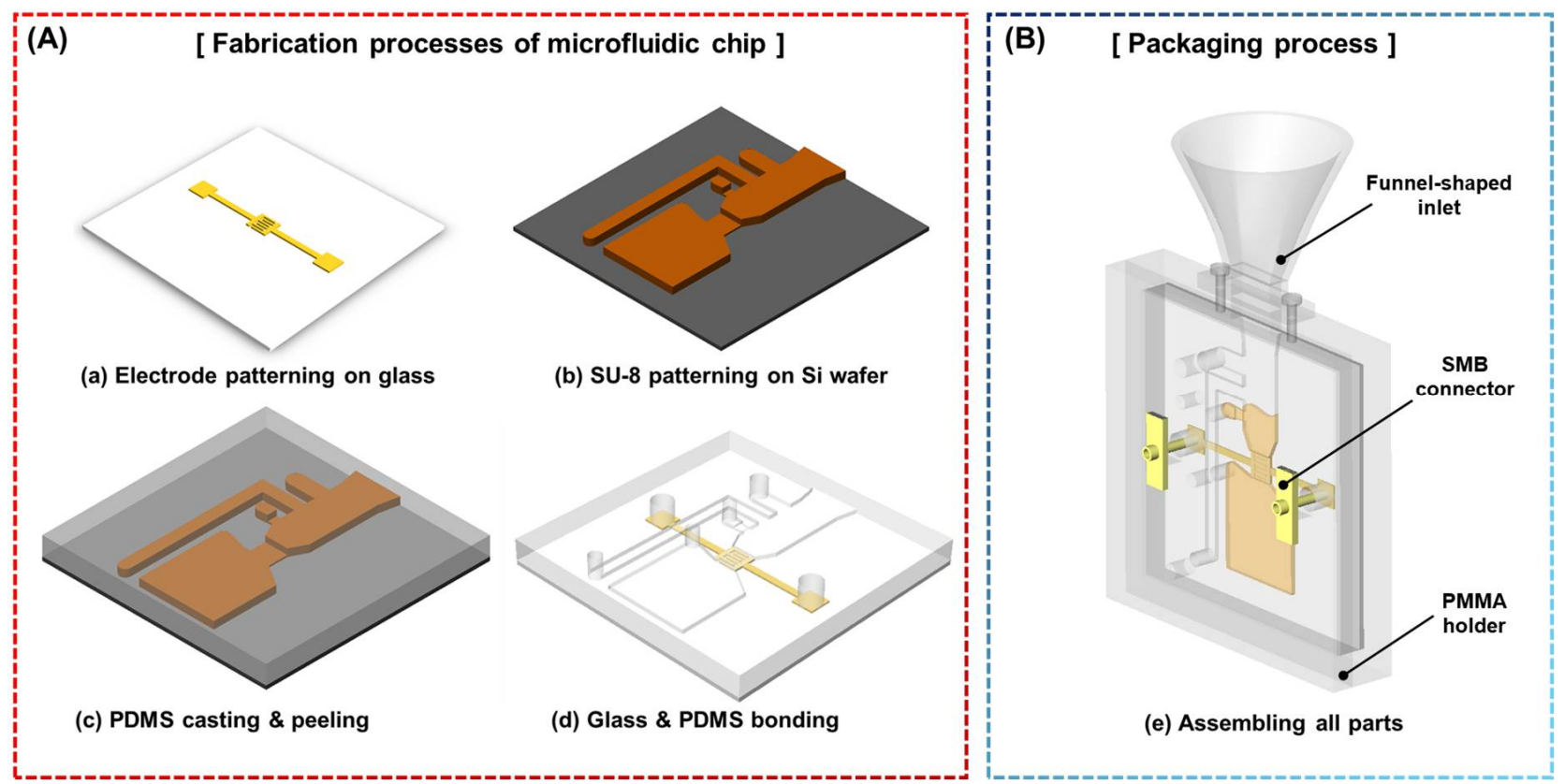

Figure 7. Simplified fabrication process of the proposed system, (A) the microfluidic MP detection chip, and (B) packaging.

In the case of packaging process (Figure 7e), the integrated microfluidic device was assembled using a polymethylmethacrylate (PMMA) holder. The rubber pad was laid for electrical insulation under the microchip covered with a case. The funnel-shaped inlet connected to the side was fabricated using a 3D printer in a single printout. SMB connectors for electrical connections, such as excitation and signal processing, were mounted on the case to fit the electrode pads.

\section{Experiments}

\subsection{Preliminary Validation Test of Particle Detection Element with Optical Synchronization}

The particle detection element is a crucial component for monitoring the delivered particles in microcirculation. Preliminary validation tests were implemented to characterize the performance of the element. In this experiment, a microfluidic rectangular sample chip ( $52.5 \mathrm{~mm} \times 52.5 \mathrm{~mm} \times 7 \mathrm{~mm}$ ) that was embedded was used. The dimensions of the microchannel were identical to those of the detection region of the integrated system.

The particle samples used in the experiment were aluminum particles (diameters of 10, 20, and $40 \mu \mathrm{m}$, RND Korea, Gwangmyeong, Korea). There were classified by size by using a sieve (10, 20, and $40 \mu \mathrm{m}$ Pluristrainer, Pluriselect, Leipzig, Germany), and $3 \mathrm{mg}$ was extracted using a microbalance (Precision balances WTC, Radwag, Radom, Poland). They were placed in a $50 \mathrm{~mL}$ centrifuge tube filled with hydraulic oil. The particle solutions were stirred for 5 min using a mixer (Maxshake-VM30, Daihan Scientific, Wonju, Korea). They were later drawn using a $5 \mathrm{~mL}$ syringe (Syringe Luer tip, Norm-Jet, Tuttlinger, Germany).

The test chip was connected to an LCR meter (E4980A, Agilent Technologies, Santa Clara, CA, USA) and the capacitance response was monitored using data processing software (LabVIEW, National Instruments, Austin, TX, USA), with the excitation voltage $\left(\mathrm{V}_{\mathrm{ac}}\right)$ and data sampling rate set to $15 \mathrm{~V}$ and $20 \mathrm{~ms}$, respectively. To demonstrate that the signals were directly generated by particle inflow simultaneously, the detection region was observed using an optical microscope (UM-12, Vitiny, Kaohsiung, Taiwan). The particle solution was loaded using a syringe pump (KD-100, KD Scientific, Seoul, Korea) at a flow rate of $10 \mu \mathrm{L} \cdot \mathrm{min}^{-1}$.

Firstly, the test was performed in clean oil conditions without particle particles to ensure a noise floor level. Figure 8 presents the capacitance signal results for the clean oil conditions. Outlier signals, such as a peak signal, were not detected. The result shows a 
stable noise floor level, and a peak signal is not generated where the particle did not exist in the detection region.

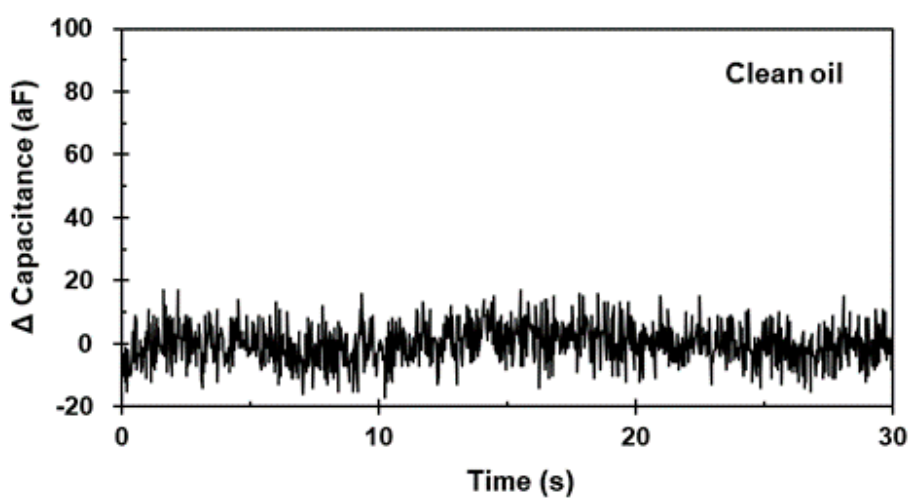

Figure 8. Measured capacitance signals for clean oil conditions.

As shown in Figure 9a, particles passing through the IDEs with an optical microscope were recorded. Figure $9 \mathrm{~b}$ shows particle samples of different sizes (10, 20, and $40 \mu \mathrm{m}$ ) passing through the detection region. Therefore, peak signals generated by the particles can be optically validated.
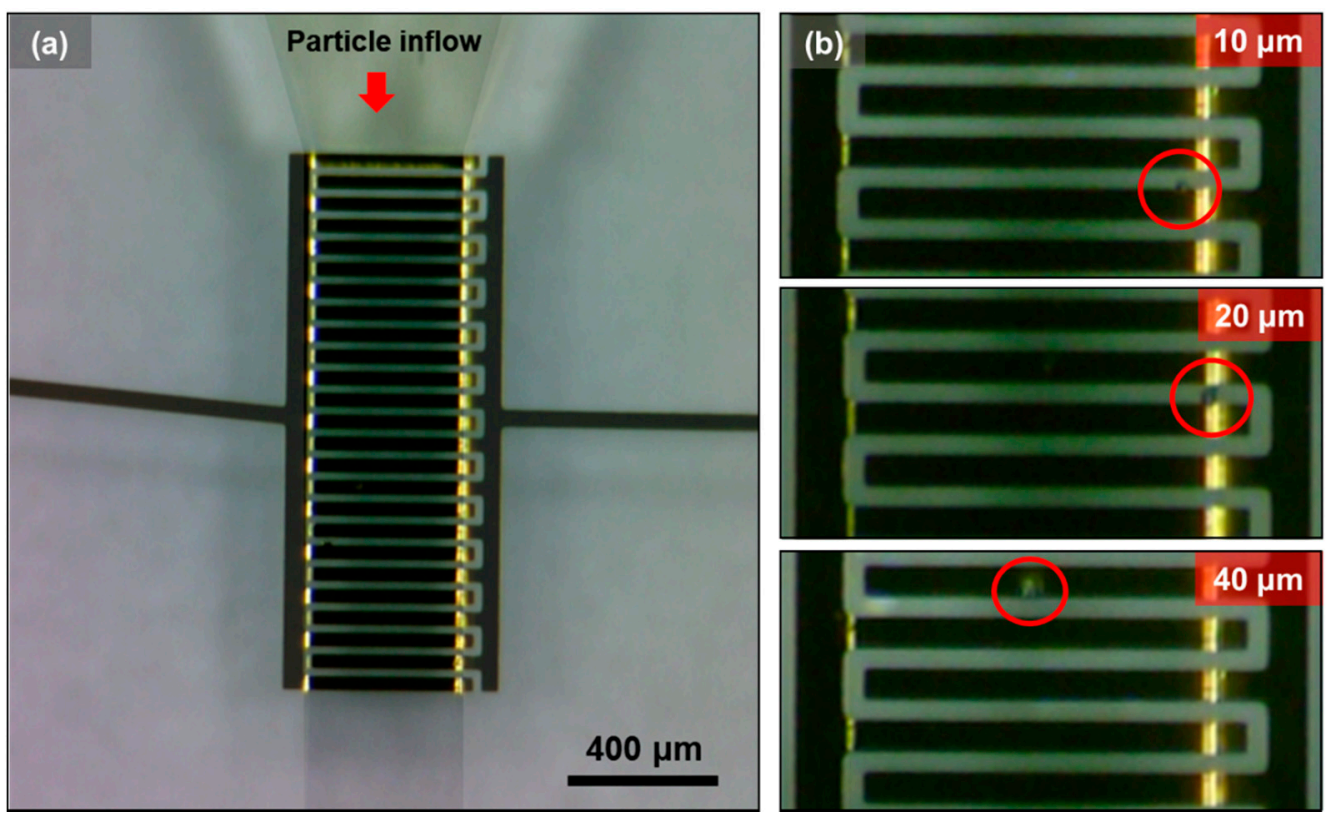

Figure 9. (a) Optical paragraph of the detection region marked with flow direction and (b) video frames of particles passing through by size $(\mathrm{d}=10,20$, and $40 \mu \mathrm{m})$.

The test was conducted by sweeping the excitation frequency, because the dielectric properties depended on the applied AC frequency. Figure 10 presents the capacitance signal results for the $20 \mu \mathrm{m}$ aluminum particles under different frequencies with $0.2 \mathrm{MHz}$ intervals. The measured capacitance signals $(\Delta C)$ generated by each particle were identified using the recorded optical synchronization. The positive pulse immediately occurred as soon as the particle crossed the detection element. The data sampling rate was appropriate for tracking the particle trajectory. The amplitudes ranged from $31 \mathrm{aF}$ to $47 \mathrm{aF}$ (Figure 10a), $30 \mathrm{aF}$ to $53 \mathrm{aF}$ (Figure 10b), $43 \mathrm{aF}$ to $57 \mathrm{aF}$ (Figure 10c), 39 to $67 \mathrm{aF}$ (Figure 10d), $38 \mathrm{aF}$ to $66 \mathrm{aF}$ (Figure 10e), and $31 \mathrm{aF}$ to $53 \mathrm{aF}$ (Figure 10f). 
(a)

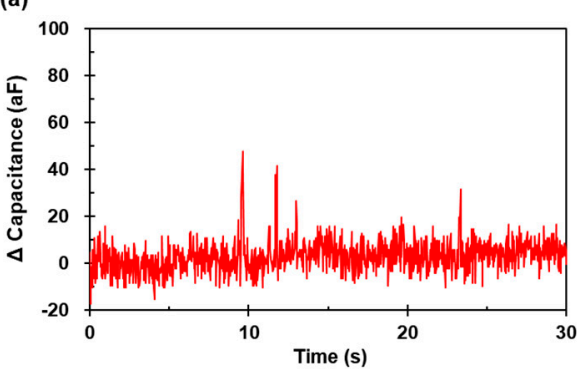

(d)

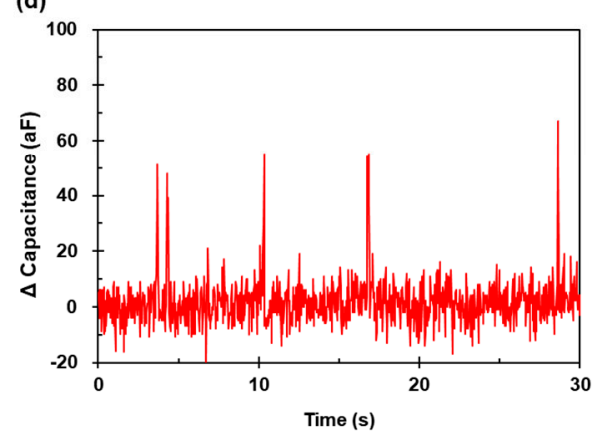

(b)

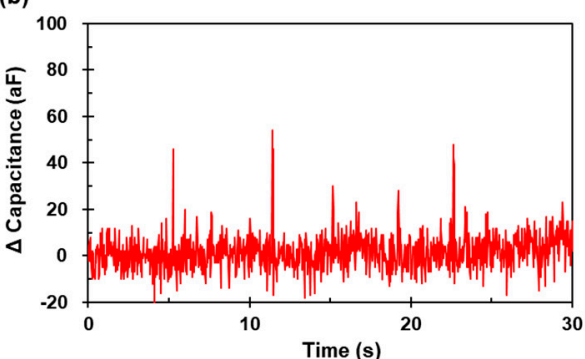

(e)

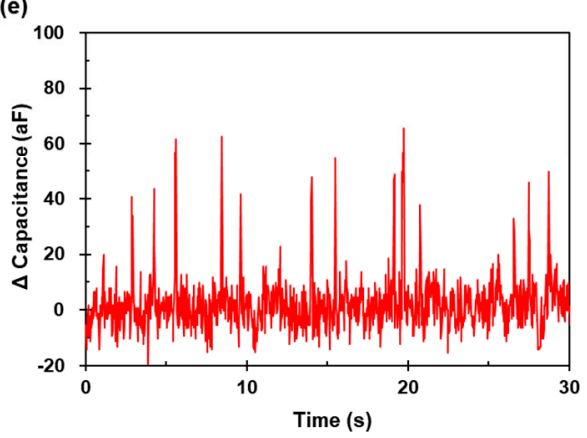

(c)

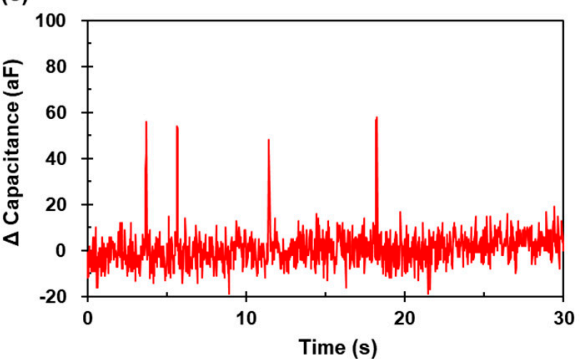

(f)

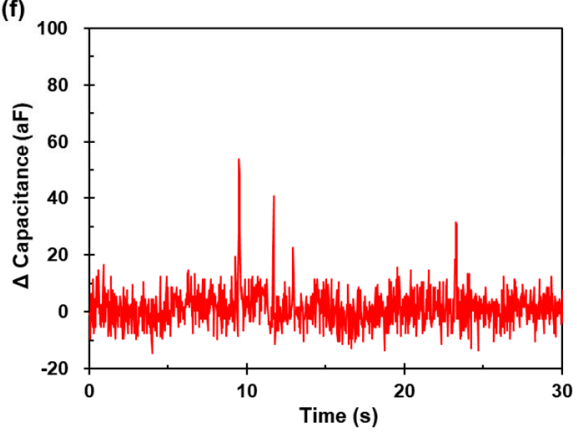

Figure 10. Measured capacitance signals for the aluminum particles $(\mathrm{d}=20 \mu \mathrm{m})$ under frequency of (a) $1.0 \mathrm{MHz}$, (b) $1.2 \mathrm{MHz}$, (c) $1.4 \mathrm{MHz}$, (d) $1.6 \mathrm{MHz}$, (e) $1.8 \mathrm{MHz}$, and (f) $2.0 \mathrm{MHz}$.

The root-mean-square noise $\left(\mathrm{N}_{\mathrm{rms}}\right)$ decreased from $6.53 \mathrm{aF}$ to $5.63 \mathrm{aF}$ depending on the frequency increment. To find the resonant frequency that increased the change in capacitance, the signal-to-noise ratio (SNR) was used, which can be expressed as

$$
\mathrm{SNR}=10 \cdot \log _{10}\left(\frac{\overline{\mathrm{C}}}{\mathrm{N}_{\mathrm{rms}}}\right)
$$

where $\bar{C}$ is the average capacitance. Based on the $20 \mu \mathrm{m}$ aluminum particle detection results, the SNR at $1.8 \mathrm{MHz}$ was the highest at 9.89 compared to the other. Further, tests were performed at this frequency for particles of smaller $(\mathrm{d}=10 \mu \mathrm{m})$ and larger $(\mathrm{d}=40 \mu \mathrm{m})$ sizes to determine the amplitude tendency.

Figure 11 presents the capacitance signal results for the $10 \mu \mathrm{m}$ and $40 \mu \mathrm{m}$ aluminum particles at $1.8 \mathrm{MHz}$. The amplitude ranges from 20 to $37 \mathrm{aF}$ (Figure 11a) and 30 aF to $142 \mathrm{aF}$ (Figure 11b), respectively. The results indicate that the particle detection element could detect individual particle sizes from $10 \mu \mathrm{m}$ to $40 \mu \mathrm{m}$. As the data sampling point was at least four, even for different particle sizes, the operational conditions were adequately determined, as presented in Figure 12.

(a)

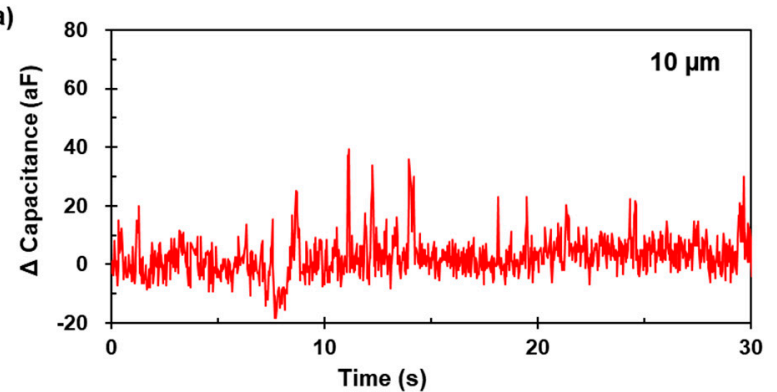

(b)

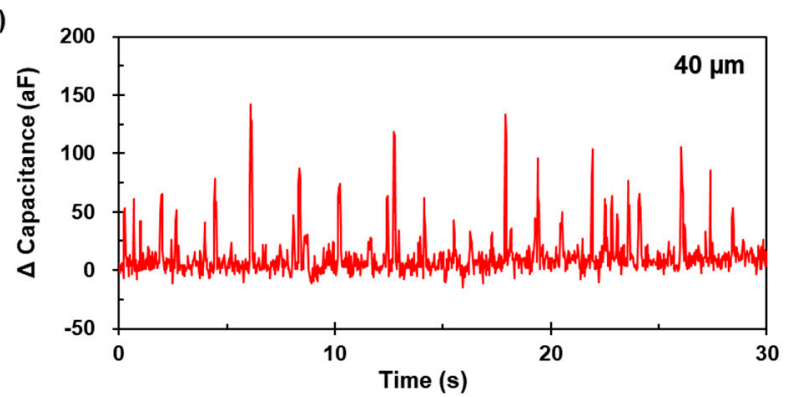

Figure 11. Measured capacitance signals for the aluminum particle sizes in (a) $\mathrm{d}=10 \mu \mathrm{m}(\mathbf{b}) \mathrm{d}=40 \mu \mathrm{m}$ at $1.8 \mathrm{MHz}$. 
(a)

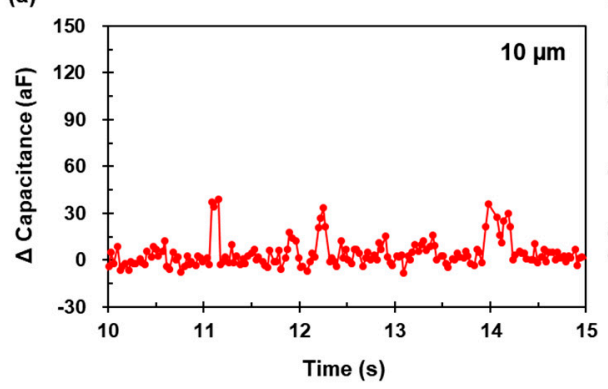

(b)

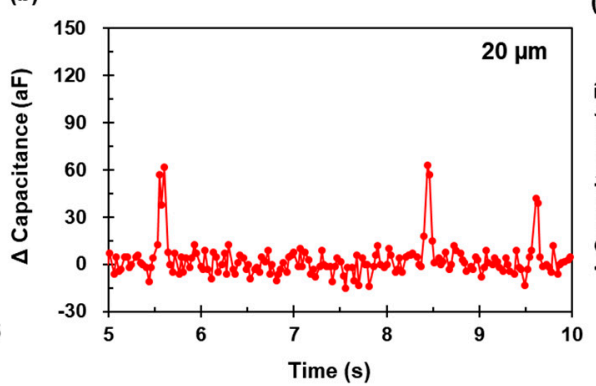

(c)

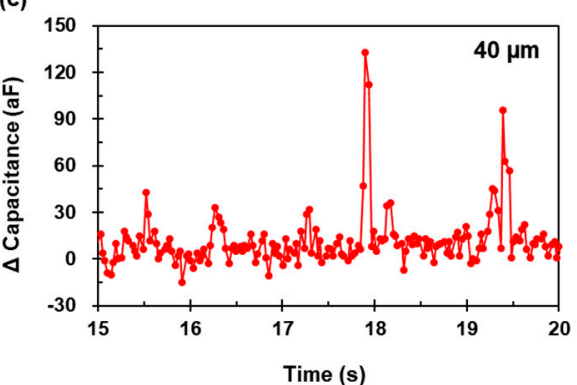

Figure 12. Close-up view of $5 \mathrm{~s}$ interval from measured capacitance signals for the aluminum particle sizes in $(\mathbf{a}) \mathrm{d}=10 \mu \mathrm{m}$ (b) $\mathrm{d}=20 \mu \mathrm{m}(\mathbf{c}) \mathrm{d}=40 \mu \mathrm{m}$ at $1.8 \mathrm{MHz}$.

From the above results, the capacitance change tends to be proportional to the particles. This is because the volume occupied by the particle is directly related to the capacitance change. Of course, the range of the measured signal amplitude based on the particle size is wide. This is because of the influence of particle $z$-axis position from the IDEs, which have commonly been pointed out at the planar electrode. Additionally, the existence of particles smaller than provided added uncertainty in that one cannot know the exact particle size. Therefore, the relationship between the particle size and the capacitance change could be possible to estimate roughly, but accuracy is still limited.

The determined geometrical structure of the detection region and the element exhibited good synergies in terms of sensing sensitivity and particle delivery. The element can detect small-sized $(\mathrm{d}=10 \mu \mathrm{m})$ MPs using low-conductive media (oil) and the megahertz level of excitation frequency. Although the relationship between the particle size and the measured capacitance signal was not accurately derived due to particle $z$-axis position and inexact particle size, the tendency was identified. The detection element can be expected to achieve airborne MP detection using similar operating conditions of the test after being integrated.

\subsection{Performance of Integrated Microfluidic Airborne MP Detection Chip}

\subsubsection{Microcirculation}

Because our proposed system aims to perform automated and continuous airborne particle collection for detection, it must be ensured that the oil microcirculation is stable. If a mismatch occurs in the flow rate of microcirculation owing to a bottleneck, overflow occurs and causes the chip to fail. It is essential to monitor the circulation under an optical microscope. Because the validated particle detection element is also sensitive to measuring the fluid flux, the operating condition of the circulation can be identified in the measured capacitance.

The proposed system was connected to a pump system comprising a miniature vacuum pump (G6/01-K-LC, Thomas, Memmingen, Germany) and a peristaltic pump (9QX, Boxer, Ottobeuren, Germany). The microcirculation rate was the same as that used in the validation test $\left(10 \mu \mathrm{L} \cdot \mathrm{min}^{-1}\right)$. The vacuum air pump used was $3 \mathrm{~L} \cdot \mathrm{min}^{-1}$. To visually confirm microcirculation, the region of the liquid surface was recorded.

Figure 13a presents the measured capacitance signals during the operation of the pump system for an hour. The periodic pattern in which the drifting signal descended and recovered was identified as $\pm 10 \mathrm{aF}$, as illustrated in Figure 13b. In addition, the surface level of the oil fluctuated, as illustrated in Figure 13c. This can be explained as a pump pulsation that occurred owing to the operation principle of the peristaltic pump. Squeeze (oil suction from the oil reservoir) for self-priming and rotation (oil supply to the oil inlet port) to force the oil onward by three rollers in the pump caused the internal pressure to increase and dissolve. Therefore, as the sensing volume was directly related to the signal, it expanded and contracted repeatedly owing to the PDMS elasticity. 
(a)

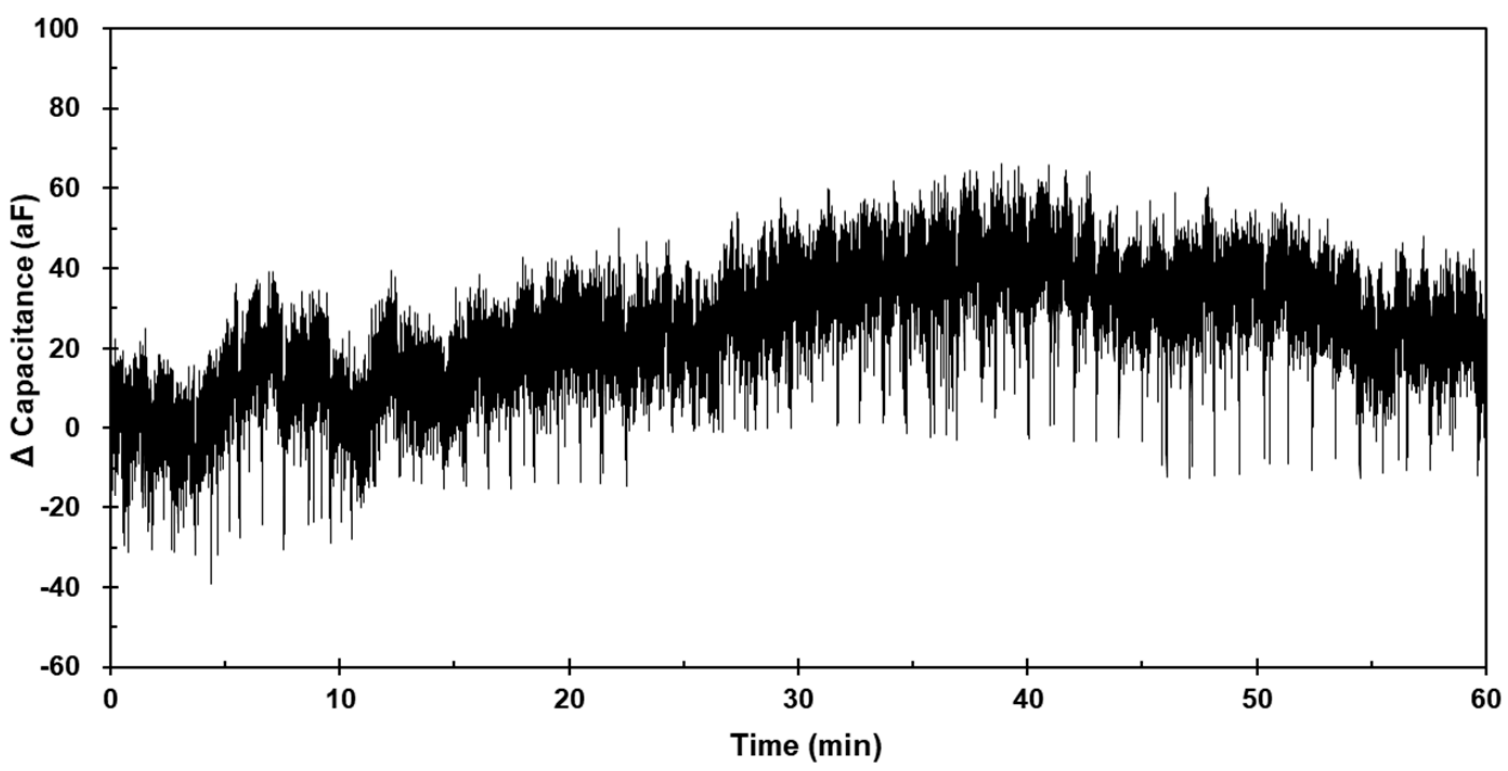

(b)

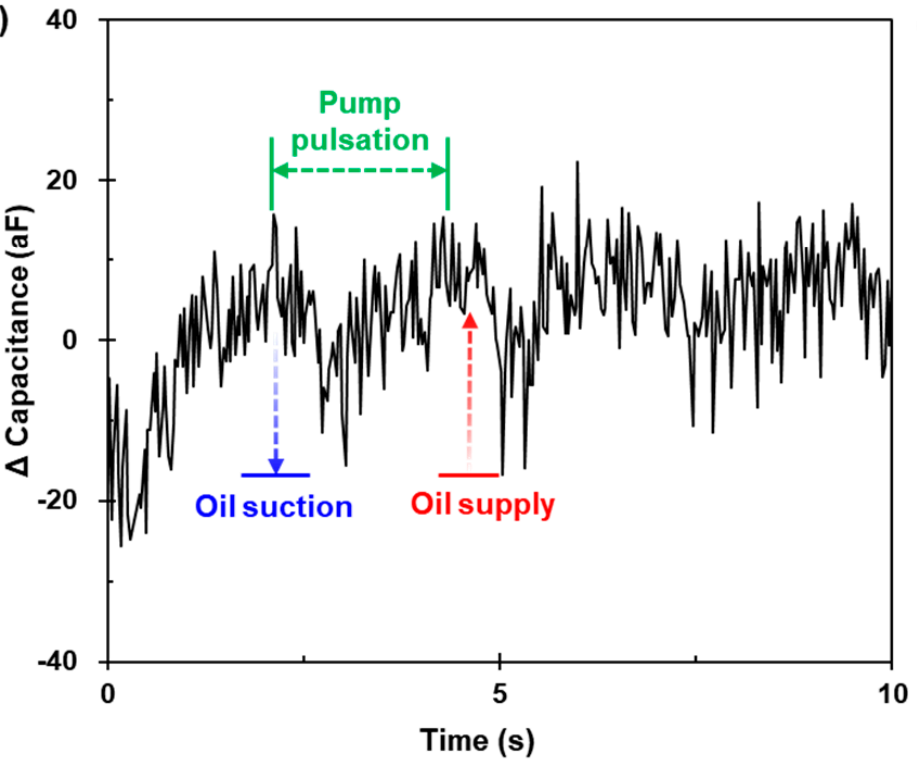

(c)

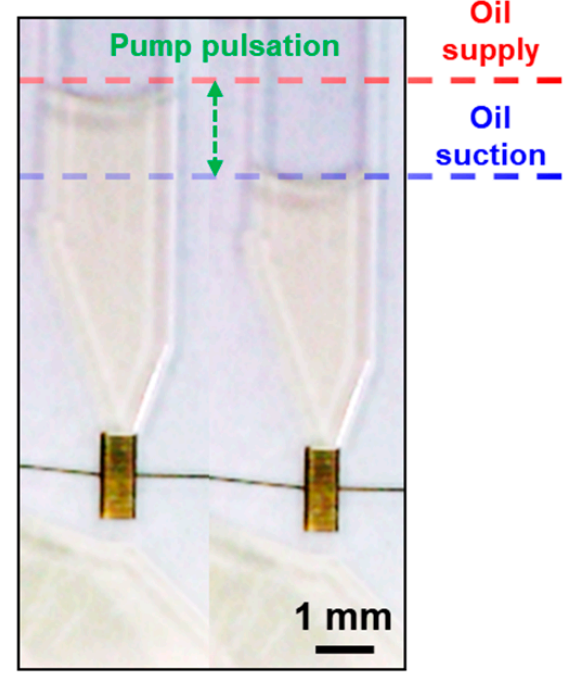

Figure 13. (a) Measured capacitance signals during operating the pump system for $60 \mathrm{~min}$, (b) close-up view of $10 \mathrm{~s}$ interval, and (c) video frames showing the fluctuating liquid interface according to peristaltic pump operation.

These results demonstrate that the microcirculation in our microfluidic system operated stably. Although periodic patterns and signal drifting are confirmed, owing to pulsation, the impact is negligible for microcirculation in the microfluidic circuit. This is because the signal of the particles delivered by the microcirculation is discernible, compared to the typical amplitude of particle detection $(\Delta C \geq 30 \mathrm{aF})$. The proposed system maintains the liquid surface for collecting airborne particles based on the recorded image of the liquid surface.

\subsubsection{Real-Time and Continuous Monitoring of Airborne MPs on a Test-Bed Chamber}

The proposed system should be capable of responding to MPs generated in the air. Consequently, the performance of particle delivery from air to oil and real-time detection based on microcirculation must be continuous and sensitive. To evaluate the performance of the proposed system in an environment where particles are generated locally in the air, the experimental setup was established in four sections: (a) the particle generation system, (b) the pump system, (c) the test-bed chamber, and (d) the performance analysis system, 
as illustrated in Figure 14. Impurities (oil droplets, moisture, and unknown particles) in the compressed air were filtered through an oil trap, diffusion dryer, and high-efficiency particulate air (HEPA) filter. The mass flow controller (VIC-D200, MKP Co., Hwaseong, Korea) adjusted the flow rate of the purified air and supplied it to a solid particle generator (model SAG 410, TOPAS, Dresden, Germany). Airborne MPs (diameter of $40 \mu \mathrm{m}$ ) were then generated as the minimum conditions by the SAG. The instrument was connected to a dilution bridge to prevent particle overflow to the chip. The diluted particles transported by the air were injected into a $0.125 \mathrm{~m}^{3}$ test-bed chamber. In the test-bed environment, the device was connected to the pump system and a performance analysis system and then evaluated.

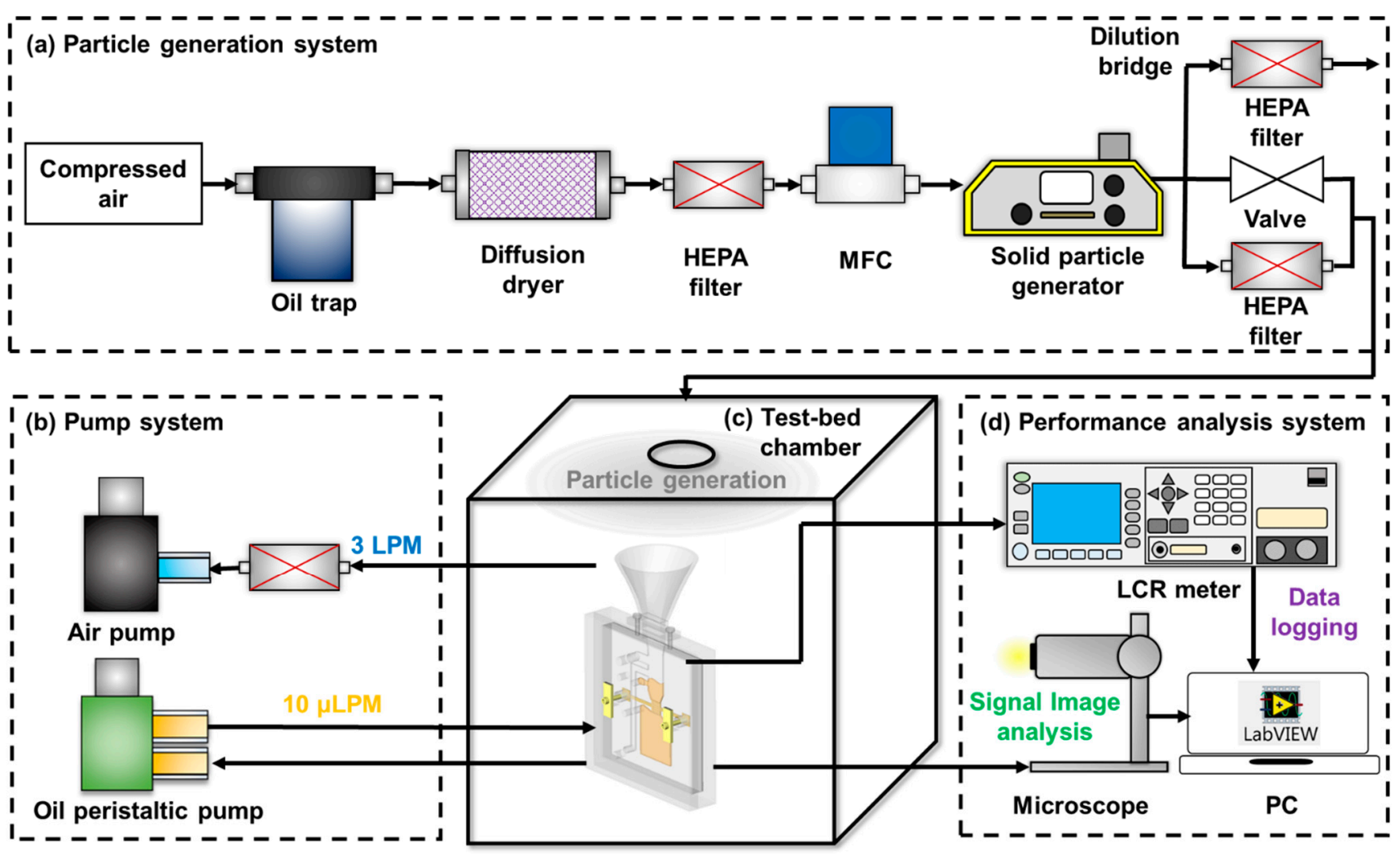

Figure 14. Schematic of the experimental set-up for performance evaluation of the proposed system; (a) particle generation system, (b) pump system, (c) test-bed chamber, and (d) performance analysis system.

Figure 15 illustrates the measured capacitance signal results for airborne MPs over $10 \mathrm{~min}$. Particle generation was performed after no pulses were applied. At the beginning of the test, until $60 \mathrm{~s}$ without particle generation, there was no visible signal compared to the baseline level $(\sim 20 \mathrm{aF})$. Within a few seconds after particle generation, pulse signals rapidly occurred. The amplitudes ranged from $30 \mathrm{aF}$ to $188 \mathrm{aF}$. After each particle generation event ( $3 \mathrm{~s}$ and $6 \mathrm{~s}$, respectively), the proposed system could be restored to the particle zeroing conditions by purging the oil reservoir with microcirculation. Typical signals $(\geq 130 \mathrm{aF}$ ) were detected, owing to the particle delivery mechanism, that is, particle collection and delivery in sequence. The reason could be considered to be particle $z$-axis position or coincidence error; multiple particles were counted as one $(d \geq 40 \mu \mathrm{m})$ without being distinguished. 

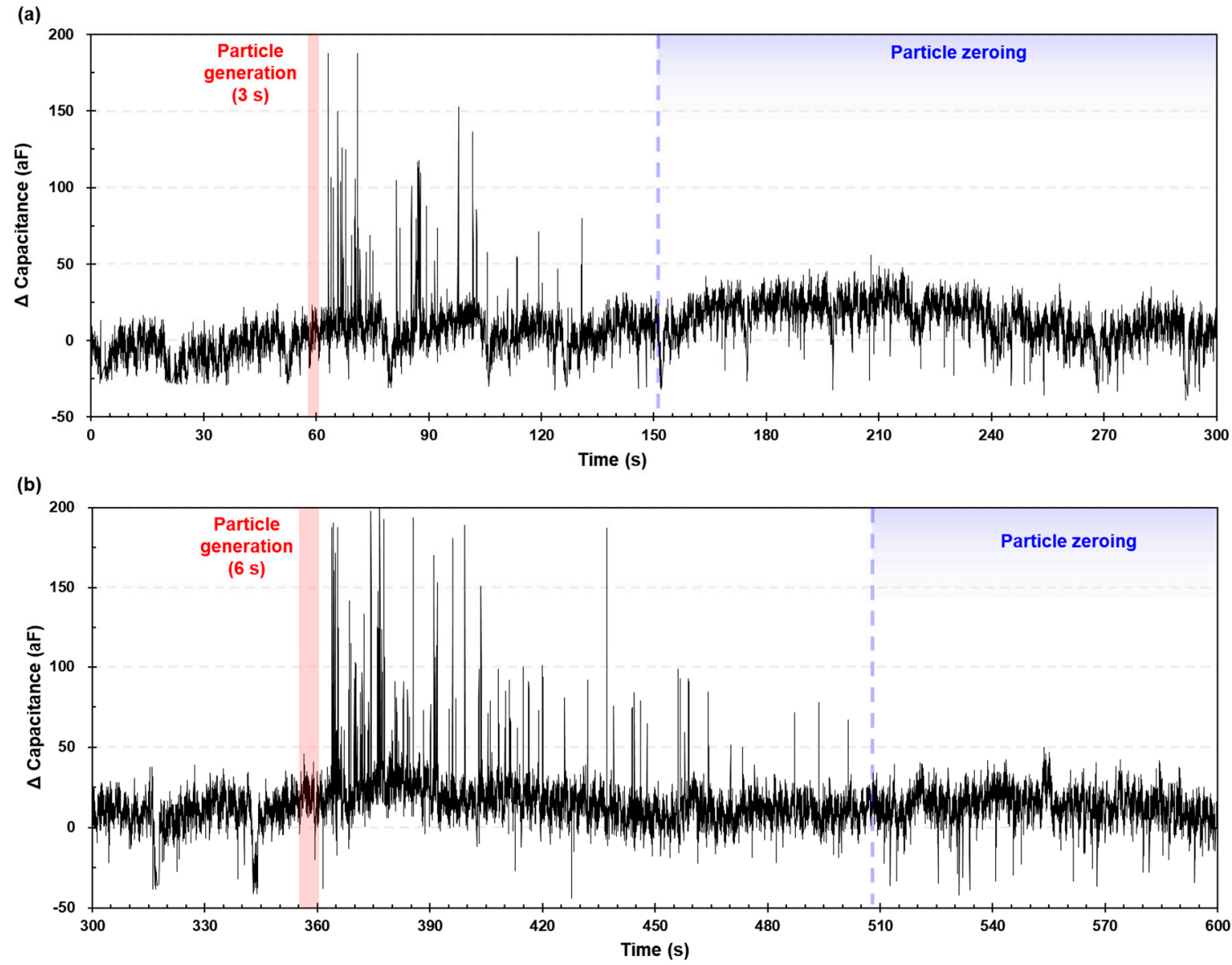

Figure 15. Performance evaluation of the proposed system in a test-bed condition; (a) $3 \mathrm{~s}$ and (b) $6 \mathrm{~s}$.

The video frames show the microcirculation-based particle delivery performance, as presented in Figure 16. At the entrance of the detection region (Figure 16a), the particles that were sampled from the air to the liquid were delivered to the particle detection region. The measured particles escaped rapidly from the detection region and sunk to the bottom by losing their inertia (Figure 16b). Subsequently, the particles were seen to be sinking and stacking at the bottom of the oil reservoir (Figure 16c).
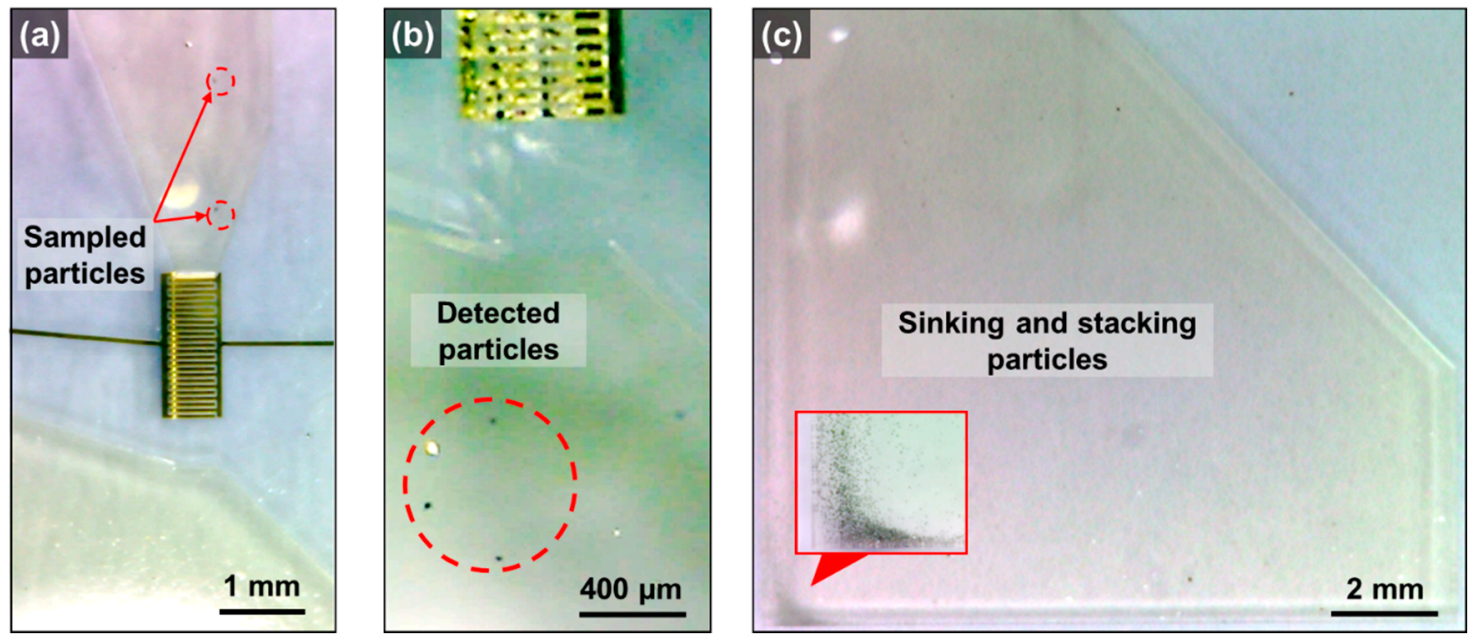

Figure 16. Video frames of particle delivery on the proposed system; (a) delivered particles at the inlet of the detection region, (b) detected particles at the outlet of the detection region, and (c) accumulated particles on the bottom of the oil reservoir. 
Figure 17 presents the number of capacitive pulses in different particle generation times. The pulses were counted based on the validated signal patterns of the individual particles. The amplitude criterion for distinguishing the noise level and pulse is $\Delta \mathrm{C} \geq 30 \mathrm{aF}$. Given the results of the validation test for the MPs in this study, this amplitude could be applied to understand the particle generation that suddenly occurred larger than a certain size. Reflecting the errors owing to particle behavior, the pulses were 39 and 84 for different particle generation times: $3 \mathrm{~s}$ and $6 \mathrm{~s}$, respectively. The longer the particle generation time, the greater the number of pulse signals identified.

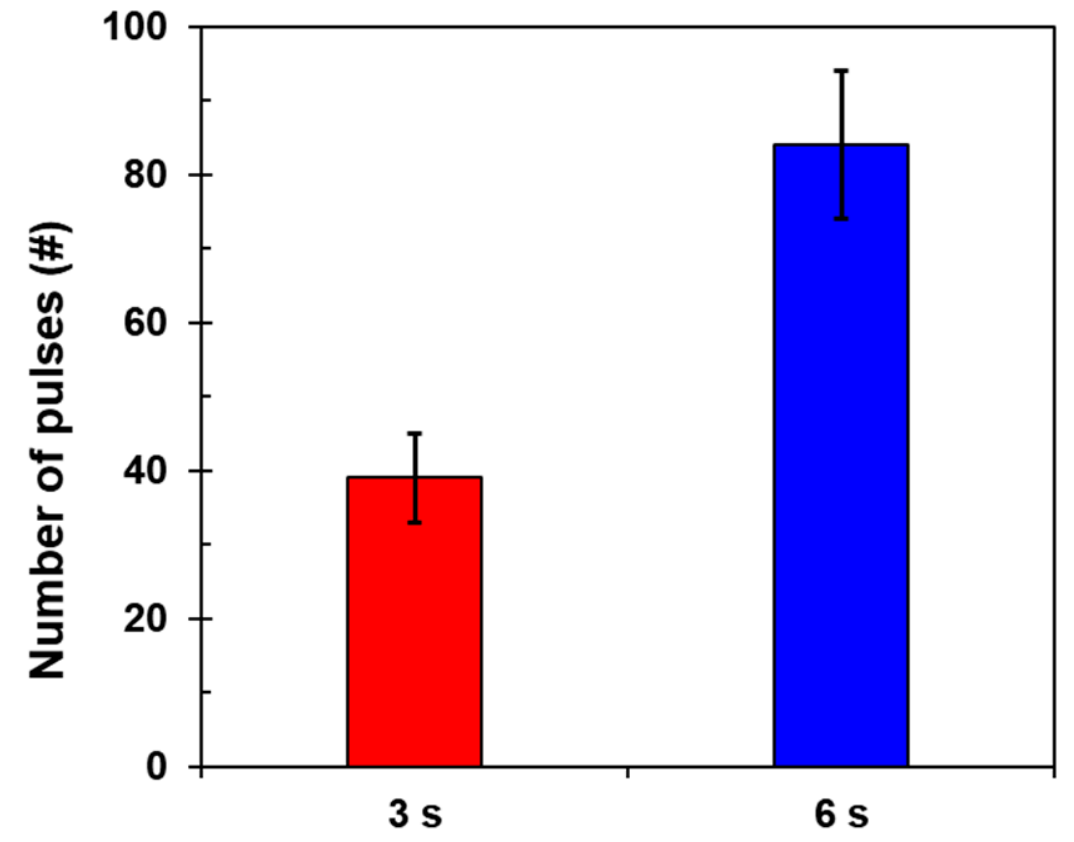

\section{Particle generation time (s)}

Figure 17. Number of capacitive pulses according to particle generation times ( $3 \mathrm{~s}$ and $6 \mathrm{~s})$.

Consequently, the proposed system can perform particle delivery and detection with continuous microcirculation. The proposed system is applicable in an environment where the MPs are generated randomly, to detect micro-sized (from $10 \mu \mathrm{m}$ to $40 \mu \mathrm{m}$ ) airborne MPs on a real-time basis. It can be used at any site where on-site airborne MP monitoring is required (e.g., semiconductor package chambers and battery manufacturing facilities).

\section{Conclusions and Discussion}

So far, many studies have been developed MEMS-compatible sensors to detect MPs. Specifically, sensors based on the inductive method have been recently developed to improve the sensitivity, in that they can distinguish ferromagnetic and non-ferromagnetic metal components [25-27]. However, they are not applicable for small-sized airborne MP detection due to their still low sensitivity to non-ferromagnetic metals. In addition, including developed capacitive sensors [28], both of them have been progressed and focused on the monitoring of oil contamination, not metal emission in the air. For our sensor, small-sized airborne MPs are monitored in real-time with continuous particle delivery and detection without periodical cleaning. A comparison between the current work with other MEMS-compatible MP detection sensors is summarized in Table 2. 
Table 2. Comparison of current work with other MEMS-compatible MP detection sensors recently developed.

\begin{tabular}{cccc}
\hline Detection Method & $\begin{array}{c}\text { Detectable } \\
\text { MP Range }\end{array}$ & Advantages & Airborne \\
MP Detection
\end{tabular}

A novel microfluidic airborne MP detection system was developed and demonstrated in this study. Owing to chip operation combining microcirculation-based particle-to-liquid collection with a capacitive sensing method onto the microfluidic platform, the proposed system could continuously monitor airborne MPs. The integrated microfluidic chip was realized on a single chip using a simple semiconductor fabrication process. Therefore, the proposed system is cost-efficient and field-portable; it is easy to use by simply exchanging the disposable chip after contamination.

Experiments were conducted to evaluate the performance of each function (particle detection and particle delivery) and the integrated system. The results indicated that the particle detection element can count the individual micro-sized MPs (at least $10 \mu \mathrm{m}$ in diameter) in real-time with high sensitivity under an optimal excitation frequency $(1.8 \mathrm{MHz})$. The proposed system integrated with the validated element exhibits signal fluctuation at the baseline, while the microcirculation was operated. However, this was insignificant compared to the level of MP detection. For each particle generation event in the test-bed chamber, the results indicated that the proposed system could detect airborne MPs in real-time and can be optically identified. Furthermore, the system can maintain continuous measurement conditions by purging itself for a while to respond to newly generated particle events. Therefore, the proposed system is capable of real-time and continuous monitoring. Herein, our sensor has limitations since it based on the microfluidic platform. Since the suction area is small and a high flow rate of vacuum air hampers the chip operation, it has the limitations on collecting a large number of airborne MPs. These limitations of this study are needed to be addressed in future work.

The possibility of on-site monitoring of the environment where airborne MPs are highly localized and unexpected can be realized with compactness and sensing performance. Thus, the proposed system can be further expected to be widely applicable in workplaces dealing with metal sources, because taking measures immediately and determining the causes of defect points is possible.

Author Contributions: Conceptualization, J.-S.Y., J.P. and Y.-J.K.; methodology, J.-S.Y., J.P. and Y.-J.K.; validation, J.-S.Y., J.P. and S.-J.Y.; data curation, J.-S.Y.; writing—original draft preparation, J.-S.Y.; writing-review and editing, J.-S.Y. and H.-R.A.; visualization, J.-S.Y. All authors have read and agreed to the published version of the manuscript.

Funding: This research was supported by the Climate Change Impact Minimization Technology Development program (NRF-2020M3H5A1081108), through the National Research Foundation of Korea (NRF), funded by the Korean government. (Ministry of Science and ICT (MSIT)).

Conflicts of Interest: The authors declare no conflict of interest. 


\section{References}

1. Leen, G.; Heffernan, D. Expanding automotive electronic systems. Computer 2002, 35, 88-93. [CrossRef]

2. Fagnant, D.J.; Kockelman, K. Preparing a nation for autonomous vehicles: 1 opportunities, barriers and policy recommendations for 2 capitalizing on self-driven vehicles 3. Transp. Res. 2014, 20, 167-181.

3. Abelein, U.; Lochner, H.; Hahn, D.; Straube, S. Complexity, quality and robustness-the challenges of tomorrow's automotive electronics. In Proceedings of the 2012 Design, Automation \& Test in Europe Conference \& Exhibition, Dresden, Germany, 12-16 March 2012; pp. 870-871.

4. Chen, R.; Luo, R.; Huang, Y.; Wu, F.; Li, L. Advanced high energy density secondary batteries with multi-electron reaction materials. Adv. Sci. 2016, 3, 1600051. [CrossRef]

5. Cheng, X.-B.; Yan, C.; Huang, J.-Q.; Li, P.; Zhu, L.; Zhao, L.; Zhang, Y.; Zhu, W.; Yang, S.-T.; Zhang, Q. The gap between long lifespan Li-S coin and pouch cells: The importance of lithium metal anode protection. Energy Storage Mater. 2017, 6, 18-25. [CrossRef]

6. Whittingham, M.S. History, evolution, and future status of energy storage. Proc. IEEE 2012, 100, 1518-1534. [CrossRef]

7. Martin, P.L. Electronic Failure Analysis Handbook: Techniques and Applications for Electronic and Electrical Packages, Components, and Assemblies; McGraw-Hill Education: New York, NY, USA, 1999.

8. Xian, T.S.; Jonet, H. Improvement of the near Short Failure through Foreign Particle Reduction in Package. In Proceedings of the 2015 IEEE 17th Electronics Packaging and Technology Conference (EPTC), Singapore, 2-4 December 2015; pp. 1-3.

9. Flanagan, I.; Jordan, J.; Whittington, H. Wear-debris detection and analysis techniques for lubricant-based condition monitoring. J. Phys. E Sci. Instrum. 1988, 21, 1011. [CrossRef]

10. Wang, N.; Wu, J.; Daniel, S. Failure Analysis of Intermittent Pin-to-pin Short Caused by Phosphorous Particle in Molding Compound. In Proceedings of the 2005 IEEE International Reliability Physics Symposium, San Jose, CA, USA, 17-21 April 2005; pp. 580-581.

11. Wu, B.; Yang, Y.; Liu, D.; Niu, C.; Gross, M.; Seymour, L.; Lee, H.; Le, P.M.; Vo, T.D.; Deng, Z.D.; et al. Good practices for rechargeable lithium metal batteries. J. Electrochem. Soc. 2019, 166, A4141. [CrossRef]

12. Wei, H.; Wenjian, C.; Shaoping, W.; Tomovic, M.M. Mechanical wear debris feature, detection, and diagnosis: A review. Chin. J. Aeronaut. 2018, 31, 867-882.

13. Jardine, A.K.; Lin, D.; Banjevic, D. A review on machinery diagnostics and prognostics implementing condition-based maintenance. Mech. Syst. Signal Process. 2006, 20, 1483-1510. [CrossRef]

14. Carter, S.; Fisher, A.S.; Goodall, P.S.; Hinds, M.W.; Lancaster, S.; Shore, S. Atomic spectrometry update. Industrial analysis: Metals, chemicals and advanced materials. J. Anal. At. Spectrom. 2009, 24, 1599-1656. [CrossRef]

15. SABA, C.S. Improving the wear metal detection of spectrometric oil analysis. Lubr. Eng. 1990, 46, 310-316.

16. Jabłońska, M.; Janeczek, J. Identification of industrial point sources of airborne dust particles in an urban environment by a combined mineralogical and meteorological analyses: A case study from the Upper Silesian conurbation, Poland. Atmos. Pollut. Res. 2019, 10, 980-988. [CrossRef]

17. Sato, T.; Matoba, Y. Rapid Detection and Element Identification of Fine Metal Particles for Underpinning Battery Quality. Hitachi Rev. 2016, 65, 263.

18. Goldstein, J.I.; Newbury, D.E.; Michael, J.R.; Ritchie, N.W.; Scott, J.H.J.; Joy, D.C. Scanning Electron Microscopy and X-ray Microanalysis; Springer: Berlin, Germany, 2017.

19. Chambers, K.; Arneson, M.; Waggoner, C. An on-line ferromagnetic wear debris sensor for machinery condition monitoring and failure detection. Wear 1988, 128, 325-337. [CrossRef]

20. Flanagan, I.; Jordan, J.; Whittington, H. An inductive method for estimating the composition and size of metal particles. Meas. Sci. Technol. 1990, 1, 381. [CrossRef]

21. Du, L.; Zhe, J.; Carletta, J.; Veillette, R.; Choy, F. Real-time monitoring of wear debris in lubrication oil using a microfluidic inductive Coulter counting device. Microfluid. Nanofluidics 2010, 9, 1241-1245. [CrossRef]

22. Du, L.; Zhe, J. A high throughput inductive pulse sensor for online oil debris monitoring. Tribol. Int. 2011, 44, 175-179. [CrossRef]

23. Wen, Z.; Yin, X.; Jiang, Z. Applications of electrostatic sensor for wear debris detecting in the lubricating oil. J. Inst. Eng. (India) Ser. C 2013, 94, 281-286. [CrossRef]

24. Shi, H.; Zhang, H.; Gu, C.; Zeng, L. A multi-parameter on-chip impedance sensor for the detection of particle contamination in hydraulic oil. Sens. Actuators A Phys. 2019, 293, 150-159. [CrossRef]

25. Wang, M.; Shi, H.; Zhang, H.; Huo, D.; Xie, Y.; Su, J. Improving the Detection Ability of Inductive Micro-Sensor for NonFerromagnetic Wear Debris. Micromachines 2020, 11, 1108. [CrossRef] [PubMed]

26. Zhang, H.; Shi, H.; Li, W.; Ma, L.; Zhao, X.; Xu, Z.; Wang, C.; Xie, Y.; Zhang, Y. A Novel Impedance Micro-Sensor for Metal Debris Monitoring of Hydraulic Oil. Micromachines 2021, 12, 150. [CrossRef]

27. Li, W.; Bai, C.; Wang, C.; Zhang, H.; Ilerioluwa, L.; Wang, X.; Yu, S.; Li, G. Design and Research of Inductive Oil Pollutant Detection Sensor Based on High Gradient Magnetic Field Structure. Micromachines 2021, 12, 638. [CrossRef]

28. Murali, S.; Xia, X.; Jagtiani, A.V.; Carletta, J.; Zhe, J. Capacitive Coulter counting: Detection of metal wear particles in lubricant using a microfluidic device. Smart Mater. Struct. 2009, 18. [CrossRef]

29. Paprotny, I.; Doering, F.; Solomon, P.A.; White, R.M.; Gundel, L.A. Microfabricated air-microfluidic sensor for personal monitoring of airborne particulate matter: Design, fabrication, and experimental results. Actuators A Phys. 2013, 201, 506-516. [CrossRef] 
30. Carminati, M.; Pedalà, L.; Bianchi, E.; Nason, F.; Dubini, G.; Cortelezzi, L.; Ferrari, G.; Sampietro, M. Capacitive detection of micrometric airborne particulate matter for solid-state personal air quality monitors. Actuators A Phys. 2014, 219, 80-87. [CrossRef]

31. Cho, Y.S.; Hong, S.C.; Choi, J.; Jung, J.H. Development of an automated wet-cyclone system for rapid, continuous and enriched bioaerosol sampling and its application to real-time detection. Actuators B Chem. 2019, 284, 525-533. [CrossRef]

32. Mainelis, G.; Masquelier, D.A.; Willeke, K.; Makarewicz, A.; Dzenitis, J.; Milanovich, F.P. Performance of a compact air-to-liquid aerosol collector with high concentration rate. J. Aerosol Sci. 2006, 37, 645-657. [CrossRef]

33. Min, M.; Parve, T.; Pliquett, U.F. Impedance Detection; Springer: New York, NY, USA, 2015; pp. 1338-1361. [CrossRef]

34. Agilent Technologies. Impedance Measurement Handbook; Agilent Technologies: Santa Clara, CA, USA, 2009.

35. Morgan, H.; Sun, T.; Holmes, D.; Gawad, S.; Green, N.G. Single cell dielectric spectroscopy. J. Phys. D Appl. Phys. 2006, 40, 61. [CrossRef]

36. Penin, N. Metal particle polarization. Bull. Lebedev Phys. Inst. 2009, 36, 63-67. [CrossRef]

37. Bhagat, A.A.S.; Kuntaegowdanahalli, S.S.; Papautsky, I. Inertial microfluidics for continuous particle filtration and extraction. Microfluid. Nanofluidics 2008, 7, 217-226. [CrossRef]

38. Di Carlo, D.; Irimia, D.; Tompkins, R.G.; Toner, M. Continuous inertial focusing, ordering, and separation of particles in microchannels. Proc. Natl. Acad. Sci. USA 2007, 104, 18892-18897. [CrossRef]

39. Dressaire, E.; Sauret, A. Clogging of microfluidic systems. Soft Matter 2017, 13, 37-48. [CrossRef]

40. Wee, M.M.R.; Buyong, M.R.; Majlis, B.Y. Effect of microchannel geometry in fluid flow for PDMS based device. In Proceedings of the RSM 2013 IEEE Regional Symposium on Micro and Nanoelectronics, Daerah Langkawi, Malaysia, 25-27 September 2013; pp. 391-393.

41. Cottet, J.; Kehren, A.; van Lintel, H.; Buret, F.; Frénéa-Robin, M.; Renaud, P. How to improve the sensitivity of coplanar electrodes and micro channel design in electrical impedance flow cytometry: A study. Microfluid. Nanofluidics 2019, 23, 11. [CrossRef]

42. Claudel, J.; Nadi, M.; Elmazria, O.; Kourtiche, D. An electrical model optimization for single cell flow impedance spectroscopy. Int. J. Smart Sens. Intell. Syst. 2016, 9, 526-536. [CrossRef]

43. Daguerre, H.; Solsona, M.; Cottet, J.; Gauthier, M.; Renaud, P.; Bolopion, A. Positional dependence of particles and cells in microfluidic electrical impedance flow cytometry: Origin, challenges and opportunities. Lab A Chip 2020, 20, 3665-3689. [CrossRef] [PubMed]

44. Abdul Rahman, M.S.; Mukhopadhyay, S.C.; Yu, P.-L. Novel Sensors for Food Inspection: Modelling, Fabrication and Experimentation; Novel Planar Interdigital Sensors; Springer: Cham, Switzerland; Heidelberg, Germany; New York, NY, USA; Dordrecht, The Netherlands; London, UK, 2014; pp. 11-35. [CrossRef]

45. Mohd Syaifudin, A.; Mukhopadhyay, S.; Yu, P. Modelling and fabrication of optimum structure of novel interdigital sensors for food inspection. Int. J. Numer. Model. Electron. Netw. Devices Fields 2012, 25, 64-81. [CrossRef]

46. Jun, L.Q.; bin Djaswadi, G.W.; bin Hawari, H.F.; Zakariya, M.A.B. Simulation of interdigitated electrodes (IDEs) geometry using COMSOL multiphysics. In Proceedings of the 2018 International Conference on Intelligent and Advanced System (ICIAS), Kuala Lumpur, Malaysia, 13-15 August 2018; pp. 1-6.

47. Eddings, M.A.; Johnson, M.A.; Gale, B.K. Determining the optimal PDMS-PDMS bonding technique for microfluidic devices. J. Micromech. Microeng. 2008, 18, 067001. [CrossRef] 